Draft Version April 23, 2022

Preprint typeset using LATEX style emulateapj v. 11/10/09

\title{
JET ROTATION DRIVEN BY MHD SHOCKS IN HELICAL MAGNETIC FIELDS
}

\author{
Christian FEndT \\ Max Planck Institute for Astronomy, Königstuhl 17, D-69117 Heidelberg, Germany \\ Draft version April 23, 2022
}

\begin{abstract}
In this paper we present a detailed numerical investigation of the hypothesis that a rotation of astrophysical jets can be caused by magnetohydrodynamic shocks in a helical magnetic field. Shock compression of the helical magnetic field results in a toroidal Lorentz force component which will accelerate the jet material in toroidal direction. This process transforms magnetic angular momentum (magnetic stress) carried along the jet into kinetic angular momentum (rotation). The mechanism proposed here only works in a helical magnetic field configuration. We demonstrate the feasibility of this mechanism by axisymmetric MHD simulations in $1.5 \mathrm{D}$ and $2.5 \mathrm{D}$ using the PLUTO code. In our setup the jet is injected into the ambient gas with zero kinetic angular momentum (no rotation). Different dynamical parameters for jet propagation are applied such as the jet internal Alfvén Mach number and fast magnetosonic Mach number, the density contrast of jet to ambient medium, or the external sonic Mach number of the jet. The mechanism we suggest should work for a variety of jet applications, e.g. protostellar or extragalactic jets, and internal jet shocks (jet knots) or external shocks between the jet and ambient gas (entrainment). For typical parameter values for protostellar jets, the numerically derived rotation feature looks consistent with the observations, i.e. rotational velocities of $0.1-1 \%$ of the jet bulk velocity.

Subject headings: shock waves - MHD - ISM: jets and outflows - stars: mass loss - stars: pre-main sequence galaxies: jets
\end{abstract}

\section{INTRODUCTION}

Astrophysical jets are highly collimated beams of high velocity material, observed in a variety of astronomical sources - among them young stellar objects (YSO), micro-quasars, or active galactic nuclei (AGN). Somewhat less collimated beams of comparatively lower speed are usually called outflows. The current understanding of jet formation is that these outflows are launched by magnetohydrodynamic (MHD) processes in the close vicinity of the central object - an accretion disk surrounding a protostar or a compact object (Blandford \& Payne 1982 Pudritz \& Norman 1983; Camenzind 1990; Pudritz et al. 2007 Cabrit 2007).

Over the past few years observational indication has been accumulated for axially symmetric differences in the radial velocity profile across protostellar jets and outflows. These radial velocity differences have repeatedly been interpreted as outflow rotation ${ }^{1}$. For one of the best studied examples, the micro-jet from DG Tau, the suggested rotational velocity is about $6-15 \mathrm{~km} / \mathrm{s}$ at about 0.2 arcsec or $40 \mathrm{AU}$ from the jet axis up to distances of $100 \mathrm{AU}$ from the star (Bacciotti et al. 2002).

As MHD outflows are launched from rotating sources by nature, the rotation of the outflow itself seems to be a characteristic feature of MHD jet formation models - from classic steady state models (Blandford \& Payne 1982), non-relativistic (Ouyed \& Pudritz 1997, Casse \& Keppens 2002) or recent relativistic simulations (Porth \& Fendt 2010). The transverse stability of relativistic

\footnotetext{
fendt@mpia.de

i In the following, for simplicity, we will omit such cautious wording regarding the existence of rotation in jets/outflows - being aware, however, that outflow rotation has not yet been fully confirmed
}

jets has been treated by two-component simulations by Meliani \& Keppens (2007). When jets originate in the inner part of a rapidly rotating disk, the outflow material is launched with a substantial amount of angular momentum. Magnetohydrodynamic models further imply angular momentum conservation along the streamlines of the outflow, and, thus, jet material which is persistently rotating. Quantitative estimates of launching-induced jet rotation based on steady-state MHD models seem to be in agreement with the observations (Anderson et al. 2003).

There are several arguments why this picture may not hold in general. The main argument is that the radial velocity profile is usually inferred from emission lines from shocked gas. It is therefore questionable that classical steady-state MHD models can be used to fit the velocity and angular momentum balance along the jet across these shocks. Furthermore, traditional non self-similar steady-state models of MHD jets indicate on both large initial jet opening angles and large jet radii compared to the Alfvén radius $r_{\mathrm{A}}$. In that case, as the rotational velocity decreases with $\sim 1 / r$ for $r>r_{\mathrm{A}}$, jet radii of $10^{2}-10^{3} r_{\mathrm{A}}$ would result in a negligible rotational velocity along the outer layers of a jet.

We like to note here that the outflow velocity profile itself might be explained without implementing jet rotation at all, and, thus, without assuming a MHD launching model for the outflow. (Soker 2005) suggested a model in which a thick and twisted accretion disk interacts with the outflow by entrainment. Inclination between the disk rotational axis and the jet axis would lead to a different amount of deceleration on both sides of the jet, thus visible in a velocity gradient across the jet.

In this paper, we propose a different explanation for the observationally indicated outflow rotation. The model 
combines two basic features of MHD outflows - (i) the fact that the observed emission is from shocked gas, and (ii) the jet shock compressed helical field leads to a magnetic torque which accelerates the outflow material in toroidal direction. Essentially, the jet kinetic angular momentum is retrieved from the magnetic angular momentum content of the jet across the shock. First hints of such a mechanism have been observed in numerical simulations by Kössl et al. (1990) or Uchida et al. (1992); Todo et al. (1992), but were not discussed in great detail, or even applied to observations ${ }^{2}$.

In the following, we first summarize the observational findings concerning outflow rotation in jets from young stars and then briefly mention the case of extragalactic jets. We continue by discussing the governing MHD processes involved in our model, and present results of our MHD simulations. These simulations are preliminary in the sense that our emphasis is on the dynamical evolution of the outflow. This did not yet include further physical processes such as cooling (apart from one example simulation), magnetic diffusivity, or radiative effects.

\section{OBSERVATIONAL INDICATION OF OUTFLOW ROTATION IN YOUNG STARS}

Here we summarize the observational background discussing a few typical, but different example sources where outflow rotation is observationally indicated.

\subsection{Pc-scale jet propagation}

To our knowledge, first observational hints on jet rotation were provided by Echelle spectroscopy of knots in the HH 212 jet (Davis et al. 2000). For one of the inner knots (SK1, 2000Au from the source), rotational velocities of $1.5 \mathrm{~km} / \mathrm{s}$ were derived, while other knots in the same jet/counter-jet did not follow the same sense of rotation (clockwise) in spite of the almost perfect sidesymmetry of HH 212 and the fact the sense of rotation of the central molecular disk is clockwise as well (Wiseman et al. 2001).

Davis et al. (2000) estimate the angular momentum of an inner accretion disk of $500 \mathrm{AU}$. Assuming that all that angular momentum will be removed by a jet with mass ejection rate of $10 \%$ the accretion rate they conclude that $\mathrm{HH} 212$ may rotate with about $2 \mathrm{~km} / \mathrm{s}$. If the disk angular momentum is removed by viscous transport only, the jet rotational velocity would be lower. We find this argument somewhat delusive as the high velocity jets is in fact launched close to the inner edge of the disk. Thus, the disk material which is ejected into the jet at these small radii has low specific angular momentum as it has lost most of its initial angular momentum during the accretion process from larger to smaller radii.

A corresponding search for outflow rotation in $\mathrm{HH} 212$ in $\mathrm{SiO}$ shocks (Lee et al. 2008) provided upper limits for rotational velocities only. Applying angular momentum conservation for steady-state the inferred jet launching radii can be constrained to $<1 \mathrm{AU}$. Most recent data of HH 212 gave no clear signature of jet rotation, but also cannot exclude rotation (Correia et al. 2009).

In the case of $\mathrm{HH} 211$, launching radii of $0.06-0.15 \mathrm{AU}$ were derived from possible jet rotation of $1.5 \mathrm{~km} / \mathrm{s}$ (Lee et al. 2007, 2009).

2 Observational indication of jet rotation had not been found by that time
The NGC 1333 IRAS 4A2 system is another jet source for which recent $\mathrm{SiO}$ observations indicate jet rotation by perpendicular velocity gradients (Choi et al. 2011). These features extend as far as almost $9000 \mathrm{AU}$ from the central protostar with derived rotational speeds of about $3 \mathrm{~km} / \mathrm{s}$ and in corotation with the underlying disk.

\subsection{Micro-jets from young stars}

The observed Doppler-shift of emission lines in the DG Tau micro-jet (Bacciotti et al. 2002) give symmetric radial velocity differences of $6-15 \mathrm{~km} / \mathrm{s}$ for the low-velocity jet component. It is difficult to imagine any other velocity pattern than rotation to explain these radial velocity gradient across the jet. The authors estimate the corresponding angular momentum flux in the low velocity component to $\dot{J} \simeq 3.8 \times$ $10^{-5} \mathrm{M}_{\odot \mathrm{yr}^{-1} \mathrm{AU} \mathrm{km} \mathrm{s}}{ }^{-1}$.

More examples were detected by the same group (Coffey et al. 2004), such as TH 28 and RW Aur or LkH $\alpha$. For RW Aur Woitas et al. (2005) find toroidal velocities in the range of $5-30 \mathrm{~km} / \mathrm{s}$ at distances 20 and $30 \mathrm{AU}$ away from the jet axis. Furthermore, both bipolar lobes rotate in the same direction.

Another candidate source for jet rotation is HL Tau for which Fabry-Perót interferometry has indicated a radial velocity gradient across the jet with a slope of $6 \mathrm{~km} / \mathrm{s} / \operatorname{arcsec}$ (Movsessian et al. 2007). As for to DG Tau, it is the low velocity component which shows a significant radial velocity slope while the radial velocity variation for the high velocity component is weak.

\subsection{Molecular outflows}

Indicate exists for rotation in molecular outflows as well. From sub-mm data of the bipolar outflow OriS6 Zapata et al. (2009) find bullets of in rotation of $5 \mathrm{~km} / \mathrm{s}$, a CO shell around the jet rotating with $2 \mathrm{~km} / \mathrm{s}$ (at $1000 \mathrm{AU}$ from the outflow axis), and a CO envelope at distances of more than $2000 \mathrm{AU}$ from the source still rotating with more then $0.5 \mathrm{~km} / \mathrm{s}$, and rotation observed up to $25,000 \mathrm{AU}$ distance from the source.

Another example is CB 26, a molecular outflow from a young T Tauri star (Launhardt et al. 2009). Detailed kinematic modeling accompanied by synthetic CO maps suggest rotational velocities of about $1 \mathrm{~km} / \mathrm{s} 100 \mathrm{AU}$ away from the outflow axis and at $1000 \mathrm{AU}$ away from the source. Alternative scenarios such as a precessing jet entraining material are discussed for this source and seem feasible as well.

\subsection{Magnetic field indication in protostellar jets}

In general, there is indirect indication of magnetic fields being present in protostellar jets. One argument has always been the magnetic activity of protostars showing large-scale flares on radio and X-rays and features of dipolar accretion indicating on a $\mathrm{kG}$ stellar surface field strength and also on a dipolar field distribution (see (Andre et al. 1988, Bouvier et al. 2007, Cabrit 2007)).

Concerning the large-scale field structure, there is the unique example of TTau where cyclotron radiation have been detected which is aligned with the jet outflow in this source (Ray et al. 1997). The infered field strength is in the range of a $\mathrm{G}$ which is huge compared to what is required by current modeling. 
However, recent observations of massive star forming regions have detected maser emission in jet sources which allows for a direct measurement of the magnetic field strength. One example is the detection of the 3D field structure around the protostar Ceph A HW 2 with $\mathrm{mG}$ field strengths (Vlemmings et al. 2010). Synchrotron emission indicating a $0.2 \mathrm{mG}$ field has been found related to the knots in the jet source HH80-81 (CarrascoGonzález et al. 2010).

\section{THE CASE OF EXTRAGALACTIC JETS}

Observational indication of rotation is lacking so far for extragalactic jets. In fact, we do not even have direct measurements of propagation speed for these sources. On the other hand, clear evidence exists for a helical magnetic field structure in extragalactic jets indicated e.g. by gradients in the rotation measure across the jets (e.g. Gabuzda et al. (2004); Lyutikov et al. (2005); Laing et al. (2006)).

The existence of radio synchrotron emission along these jets on time scales beyond the synchrotron cooling time requires re-heating of the jet material, most probably by internal shocks. Together, the helical field structure and the internal shocks, provide the necessary prerequisites for the acceleration mechanism discussed below. Therefore, signatures of jet rotation can also expected from these sources.

In fact, there is one jet source where azimuthal jet motion has been detected. Imaging interferometry of emission lines have detected a braided structure in the jet system of NGC 4258, which could be modeled by a triple helix of jet motion on $5-10 \mathrm{kpc}$ scale (Cecil et al. 1992). This kpc jet is aligned with a nuclear VLBA radio jet (Herrnstein et al. 1997), thereby suggesting a physical connection. However, even if the helix model would explain the physical conditions in the kpc jet, there seems to be no plausible explanation yet about the intrinsic process causing such a complex outflow structure.

In this context it is interesting to note the relevance of jet rotation for modeling the heating of relativistic jet plasma by shear and centrifugal acceleration for electrons (Rieger \& Mannheim 2002, Rieger \& Duffy 2004) or protons (Dempsey \& Rieger 2009).

\section{MHD JETS AND ROTATION}

Magnetohydrodynamic jets are launched from rotating sources. The initial acceleration is by centrifugal forces along the magnetic field lines (magneto-centrifugal). Beyond the Alfvén point along each field line, the outflow material becomes inertially so heavy that co-rotation of matter and magnetic field cannot longer be maintained. Thus, the field lines will be "bent" from a mainly poloidal into a helical geometry (a toroidal field component is induced). This allows the matter to "slide" along the field lines in toroidal direction. As a consequence, at large radii the jet material moves outwards in almost radial direction by conserving its total angular momentum. The angular velocity decreases with radius.

Magnetohydrodynamic acceleration and collimation of outflows implies transfer of magnetic energy to kinetic energy, and, likewise, transfer of kinetic angular momentum in magnetic angular momentum. The exact balance between these quantities results from the local forcebalance throughout the outflow. The solution of the equations is complex and requires semi-analytical modeling and/or numerical MHD simulations.

For understanding the considerations of this paper, it is helpful to re-call some basic properties of axisymmetric, stationary-state theory of MHD winds and jets (see e.g. Pudritz et al. (2007)). In general, steady-state MHD implies the existence of conserved quantities along the magnetic flux surfaces. In the case of axisymmetry five such quantities can be defined. The first one is the magnetic flux, $\Psi(r, z) \equiv(1 / 2 \pi) \int \vec{B}_{\mathrm{p}} d \vec{A}$, indicating that magnetic field lines lie on magnetic flux surfaces. The second one is the mass flux $\eta(\Psi) \equiv \int \rho \vec{v}_{\mathrm{p}} d \vec{A}$ per magnetic flux surface $\Psi$. The third one is Ferraro's iso-rotation parameter $\Omega_{\mathrm{F}}(\Psi) \equiv\left(v_{\mathrm{p}} B_{\phi} / B_{\mathrm{p}}-v_{\phi}\right) / r$, often interpreted as angular velocity of the field lines. The fourth one is the total (specific) angular momentum

$$
L(\Psi)=r v_{\phi}-\frac{r B_{\phi} B_{\mathrm{p}}}{4 \pi \rho v_{\mathrm{p}}} \equiv \Omega_{\mathrm{F}} r_{\mathrm{A}}^{2} .
$$

The fifth one is the total (specific) energy

$$
E(\Psi)=\frac{1}{2} v_{\mathrm{p}}^{2}+\frac{1}{2} v_{\phi}^{2}-\frac{r B_{\phi} B_{\mathrm{p}} \Omega_{\mathrm{F}}}{4 \pi \rho v_{\mathrm{p}}},
$$

where, for simplicity, we have omitted gravity and gas pressure (cold wind assumption). In steady-state theory $E(\Psi), L(\Psi)$, and $\eta(\Psi)$ are governed by the regularity condition across the fast magnetosonic point, the Alfvén point at $r=r_{\mathrm{A}}$, and the slow magnetosonic point, respectively.

Essentially, both the total energy and the total angular momentum (flux) consist of a magnetic and a kinetic contribution. A classical Blandford-Payne-type outflow is launched with $v_{\phi}>>v_{\mathrm{p}}$ and $B_{\phi}<B_{\mathrm{p}}$. Beyond the Alfvén point $v_{\phi}$ decreases and the magnetic field becomes dominated by $B_{\phi}>B_{\mathrm{p}}$. Likewise, kinetic angular momentum of the ejected jet material, which is co-rotating with the disk, is increasingly transferred to the outflow magnetic angular momentum.

The existence of conserved quantities along the field lines can be used to physically link the very inner region where the jet is launched (not yet possible to resolve observationally) to the asymptotic region of the outflow (accessible for observations). Anderson et al. (2003) have applied this formalism to the observed rotational features in the DG Tau jet (Bacciotti et al. 2002), obtaining launching radii of $0.3-4.0 \mathrm{AU}$. While this approach could be applied to the low velocity jet component, it does not fit, however, the high-velocity jet component of DG Tau. Using a similar method for the bipolar jet from RW Aur, Woitas et al. (2005) find launching radii less than $0.5 \mathrm{AU}$ and a magnetic lever arm (Alfvén radius to launching radius) of about four.

In the following we will argue that the classical stationary MHD wind approach discussed above is questionable as sole explanation of rotating outflows. In the next section we will suggest and discuss an alternative scenario. Our main argument is that radial velocity measurements of stellar jets and outflows usually consider line emission of shocked gas or molecules. For example, LavalleyFouguet et al. (2000) prove shock excitation of forbidden emission lines in DG Tau and Woitas et al. (2005) explicitely state that rotation is more evident in the for- 
bidden lines [OI] and [NII]. During shock transition, the jet material changes its dynamical state instantly. Shock transitions are not included with the classical stationary state MHD wind theory. Instead, conservation laws must be applied in form of jump conditions, and the direct connection of the observed rotating material with the disk by ideal MHD conservation laws is questionable. This argument may hold in particular for the large scale jets and outflows as e.g. for HH 212 or molecular outflows. The molecular material might also trace entrained interstellar material. In this case, it has not been launched from the central region with intrinsic angular momentum.

Early work on steady-state MHD jets has suggested that jets do expand substantially from their launching area close to the star to the asymptotic (observed) regime. Expansion factors of 100-1000 (from 0.1 AU up to $100 \mathrm{AU}$ jet diameter) have been suggested. These classical solutions predict Alfvén radii $R_{\mathrm{A}}$ of about ten foot point radii, $R_{\mathrm{A}} \simeq 10 R_{\mathrm{F}}$ and, therefore, a substantial decrease of the toroidal outflow velocities for radii $r>>R_{\mathrm{A}}$ to $v_{\phi} / v_{\text {jet }}<0.001$. This is due to the large initial opening angles which are a result from non-self-similar MHD solutions (see Fendt et al. (1995); Fendt \& Camenzind (1996), but also recent simulations of stellar wind magnetospheres by Matt et al. (2008, 2010) resulting in a similar field geometry).

We conclude that while being consistent with stationary state MHD jet formation, the formalism suggested by Andersen et al. and subsequently applied by other authors is probably not a fully realistic picture to explain the observed jet rotation.

\section{MHD SHOCKS CRANKING JETS}

In the following we discuss another framework of how jets and outflows may be set in rotation. The basic idea is that a compression of the toroidal magnetic field component in MHD shocks results in a Lorentz torque in toroidal direction which accelerates the jet material. The jet kinetic angular momentum is retrieved from the magnetic angular momentum content of the jet by the shock. This mechanism works only for helical magnetic fields. While in stationary state MHD the kinetic and magnetic angular momentum is gradually exchanged along the flux surfaces, the shock wave leads to a sudden exchange.

\subsection{Lorentz torque}

The MHD Lorentz force is $\vec{F}_{\mathrm{L}} \sim \vec{j} \times \vec{B} \sim(\nabla \times \vec{B}) \times \vec{B}$. Jet rotation can be achieved by its toroidal component

$$
\vec{F}_{\phi} \sim\left(\nabla \times \vec{B}_{\phi}\right) \times B_{\mathrm{p}} .
$$

Across the shock the toroidal field component is compressed, thus giving rise to a toroidal force component. The compression ratio itself depends on the jet dynamics. For simple geometries the field compression can be estimated by the Rankine-Hugoniot jump conditions. More complex configurations require numerical simulations to follow the shock evolution.

The magnetic flux is conserved across the shock ${ }^{3}$ $\left[B_{z}\right]_{1}^{2}=0$, but the perpendicular magnetic field component typically undergoes a change. In case of cylindrical

\footnotetext{
${ }^{3}$ Index ' 1 ' denotes the upstream region and index '2' the downstream region
}

coordinates (which we are using here), the $B_{\phi}$-component replaces the perpendicular component in Cartesian coordinates, resulting in an expression for the compression

$$
\frac{\left[B_{\phi}^{2}\right]_{1}^{2}}{B_{z}^{2}}=\frac{2 m_{z}^{2}\left\langle B_{\phi}\right\rangle^{2} / B_{z}^{2}}{m_{z}^{2}-\left(B^{2} / 4 \pi\left\langle\rho^{\star}\right\rangle\right)} \frac{\left[\rho^{\star}\right]_{1}^{2}}{\left\langle\rho^{\star}\right\rangle},
$$

(Uchida et al. 1992), where $m_{z} \equiv \rho v_{z}$ denotes the (conserved) axial momentum, $\left\langle\rho^{\star}\right\rangle \equiv 0.5\left(1 / \rho_{1}+1 / \rho_{2}\right)$ the averaged inverse density from upstream and downstream, $\left[\rho^{\star}\right]_{1}^{2}$ the difference in the inverse density upstream and downstream, and $\left\langle B_{\phi}\right\rangle^{2} \equiv\langle B\rangle^{2}-B_{z}^{2}$ the average toroidal field strength.

The dynamical structure of the propagating shock could be complex. As typically for this setup, four shocks are existent, accompanied by a contact discontinuity, each satisfying the jump conditions (see e.g. Uchida et al. (1992); Ryu \& Jones (1995)). Considering the toroidal field strength, an increase within the first MHD (fast) shock is followed by a decrease across the second MHD (slow) shock, then again by an increase in the third (slow MHD) shock, and another decrease in the fourth (fast MHD) shock (going from downstream to upstream; see below and Uchida et al. (1992); Ryu \& Jones (1995)).

A vast number of multi-dimensional jet propagation simulations have been published. Only few of them apply a helical magnetic field configuration and some of those have already provided numerical indication for toroidal velocity changes due to toroidal magnetic torques in jet shocks. A connection to or predictions of the observable jet rotation has not been made (such observations were not available in that time). Early simulations of jet propagation do not show rotation (Clarke et al. 1986) since treating a purely toroidal magnetic field only. To our knowledge, the MHD simulations by Kössl et al. (1990) were first indicating rotational velocity changes caused by a toroidal magnetic torque across a shock. Applying jet parameters such as Mach numbers of 4 , plasma- $\beta=1$, and a helical field configuration $B_{\phi} \simeq B_{\mathrm{p}}$ the rotational velocities obtained were rather small $v_{\phi}<0.1 c_{\mathrm{s}}$.

Uchida et al. (1992); Todo et al. (1992) present MHD simulations in $1.5 \mathrm{D}$ and $2.5 \mathrm{D}$ which in particular compare $1.5 \mathrm{D}$ simulations in cylindrical coordinates to analytical solutions of steady MHD shocks. In their case, the rotational velocities obtained for $M_{\mathrm{A}}=1.0,2.5,6.0 \mathrm{simu}-$ lations are comparatively high - in fact up to 10-40speed, and, thus, not realistic compared to the observed values. In fact, in a forthcoming paper on 3D simulations Todo et al. (1993) discuss such high azimuthal velocities as foremost indicator of a jet instability.

Rosen et al. (1999) investigate jet stability under the influence of helical magnetic fields, but do not discuss jet rotation in particular. Simulations by Stone \& Hardee (2000) consider both steady and pulsed MHD jets with helical magnetic field, in particular their cooling and stability properties, but again do not stress jet rotation.

\subsection{Angular momentum budget}

A basic prerequisite for the applicability of the process discussed in this paper is a sufficient amount of magnetic angular momentum available, which can be converted within the shock region. We may estimate the angular momentum budget from numerical models of the jet 
formation region which provide solutions to the MHD equations along the accelerating and expanding jet.

The amount of magnetic angular momentum flux $L_{\text {mag }}$ in respect to the kinetic angular momentum flux $L_{\text {kin }}$ depends crucially on the MHD state of the flow. Analytical estimates (Blandford \& Payne 1982, Pelletier \& Pudritz 1992 show that for sub-Alfvenic flows $L_{\mathrm{mag}}>>L_{\mathrm{kin}}$, while for marginally super-fast flows $L_{\mathrm{mag}} \simeq 2 L_{\mathrm{kin}}$. For a (poloidal) fast magnetosonic Mach number $M_{\mathrm{FM}}=2$ the kinetic angular momentum flux dominates, $L_{\mathrm{mag}} / L_{\mathrm{kin}} \simeq$ $2 / M_{\mathrm{FM}}^{2}=0.5$.

Numerical simulations of MHD jet formation from Keplerian disks show, however, that typically only the inner jet (the part close to the jet axis) becomes super-fast magnetosonic (Ouyed \& Pudritz 1997: Krasnopolsky et al. 1999; Fendt \& Cemeljic 2002, Fendt 2006; Porth \& Fendt 2010). This suggests an asymptotic jet structure consisting of a narrow core dominated by kinetic angular momentum, which is wrapped by an envelope dominated by magnetic angular momentum flux. It is this envelope around the core jet, which i) (shock-) interacts with the ambient medium, and ii) is accessible to the observations. Thus, for the purpose of this paper it is convenient to assume that a substantial (i.e. sufficient) amount of magnetic angular momentum is present in the asymptotic jet.

Numerical simulations of MHD jet formation from Keplerian disks also show quite a variety in the asymptotic jet outflow they produce (and which is subject to the present paper). We refer to our past work (Fendt 2006) which in very detail investigated how the launching conditions as the disk magnetic field profile and strength and also the mass flux distribution affects the asymtotic flow. In particular the radial profile of the disk wind magnetization (steep or flat) determines the collimation of the outflow (weakly, resp. strongly collimated). We therefore decided to scan a rather large parameter range in the typical jet characteristics as Mach number or plasma beta.

Recently Ramsey \& Clarke (2011) have performed jet formation simulations spanning from the disk surface well into the asymptotic regime, enclosing a huge, yet unprecedented area of $256 \times 4096$ AU size. These simulations show i) that a well ordered, global field distribution remains present also for the most distant outflow regime, ii) that the asymptotic flows launched with different initial plasma- $\beta$ tend to approach asymptotic states with average plasma- $\beta$ close to unity (although there is still quite a spread during the simulation time scales considered), and iii) that flows of different initial plasma- $\beta$ may reach quite different asymptotic states concerning mass fluxes of velocities.

In summery, we decided to run our simulations for a variety of leading jet parameters, considering also the fact that jets from protostars and AGN could be intrinsically different.

\section{MODEL SETUP FOR THE MHD SIMULATIONS}

In order to quantify the toroidal torque in jets and the subsequent toroidal velocity structure, we perform MHD simulations of jet propagation applying different flow geometries, magnetic field distributions, and jet dynamical parameters. In order to demonstrate the efficiency of the proposed mechanism, we inject the jet material non-
Table 1

Typical astrophysical scaling parameters for jets from young stars and AGN

\begin{tabular}{ccc}
\hline & Stellar jets & AGN jets \\
\hline$v_{\text {jet }}$ & $\simeq 300 \mathrm{~km} / \mathrm{s}$ & $\simeq 0.3 c$ \\
$\rho_{\text {jet }}$ & $\simeq 10^{4} \mathrm{~cm}^{-3}$ & $\simeq 10^{-4} \mathrm{~cm}^{-3}$ \\
$B_{\text {p,jet }}$ & $10-100 \mu \mathrm{G}$ & $10-100 \mathrm{G}$ \\
\hline
\end{tabular}

rotating, thus with vanishing kinetic angular momentum.

We use the PLUTO code (Mignone et al. 2007) to solve the standard set of ideal MHD equations. We apply an ideal equation of state with polytropic index $\gamma=5 / 3$. We also discuss one example simulation for which cooling is considered.

In order to re-scale normalized code variables to astrophysical magnitudes, one may apply typical numbers values for protostellar jets or AGN jets as shown in Tab. 1

\subsection{Simulations in $1.5 \mathrm{D}$}

With "1.5D" we denote axisymmetric simulations taking into account all three spatial coordinates of the vector fields, however, putting one of the vector field component to zero initially 4 . We also limit the grid extension to only one cell in this direction.

The general setup is that of a typical MHD shock tube test simulation. High-density/high pressure gas of high velocity is continuously injected from the left-hand side of the box (considered as the jet flow). Low-density/low pressure material is placed at the right-hand side of the box (considered as the ambient gas) and is allowed to move out of the box. With this setup we extend previously existing simulations (Uchida et al. 1992, Ryu \& Jones 1995) to the specific question of jet rotation and for parameter studies of that.

We apply different magnetic field configurations. For the simulations in cylindrical coordinates we assume an initial axial field $B_{z}(z)$ which is constant along the grid. The initial toroidal field $B_{\phi}(z)$ is either only present on the left side (upstream) or constant along the grid. For comparison with the literature and in order to test the 1.5 D approach in cylindrical coordinates, we have computed the same problem also in Cartesian coordinates and have found perfect agreement (see appendix).

The numerical resolution varies from 5000 - 20000 grid cells for 20 physical scale lengths.

\subsection{Simulations in 2.5D}

With "2.5D" we denote axisymmetric simulations taking into account all three vector components. By definition the simulations sustain axisymmetry as the $\phi$ derivatives are not considered in this option of the code. A jet of unity radius is injected into a box of ambient gas with constant density, pressure, and longitudinal magnetic field. We apply a non-equidistant grid with high resolution across the jet beam and the interaction region

4 That is the $r$-component in cylindrical coordinates. We have also performed comparison simulations in Cartesian coordinates with an initially vanishing $x$-component. For both cases we have approved that these components remain negligible during the simulation, i.e. $<10^{-14}$. 
with the ambient gas $(r<1.5,100$ cells $)$, and a somewhat lower resolution in the ambient gas $(1.5<r<7.0$, 200 cells).

It would be interesting to extend the present investigation to the $3 \mathrm{D}$ case in a future paper. Although the principle mechanism proposed here will be the same in the 3D case, the overall jet structure could be different and also the characteristic instabilities affecting the jet flow. In particular large-scale instabilities as the $m=1$ kink mode could appear and disturb the jet flow. Such instabilities would develop beyond the Alfvén surface (thus in the super-Alfénic flow), and may destroy the large-scale helical field structure (Moll 2009). This is a serious argument, however, it affects the standard model of jet rotation (e.g. Anderson et al. (2003)) even more. On the other hand 3D simulations of supersonic have revealed a quite similar general structure compared to the $2 \mathrm{D}$ case (O'Neill et al. 2005). Also, 3D simulations of jet formation by Ouyed et al. (2003) revealed a self-stabilizing ability of the jet due to its axial spine. We finally note the fact that we do observe large-scale collimated outflows which tells us that jet stability must be maintained by some (yet unknown) process.

\subsection{Main simulation parameters}

Our simulations are characterized by the following set of parameters.

We apply different jet densities $\rho_{\text {jet }}$, while the initial ambient density is always the same, $\rho_{\text {ext }}=1.0$. The jet inflow velocity is $v_{\text {jet }}$. In the case of $2.5 \mathrm{D}$ simulations this is the maximum jet velocity as we prescribe a cosine profile for the injection speed across the jet inlet. The ambient gas is initially at rest. The jet gas pressure is $P_{\text {jet }}$ and the ambient gas pressure is $P_{\text {ext }}$. The strength of the magnetic field is parameterized by the (squared) jet internal poloidal Alfvén Mach number,

$$
M_{\mathrm{Ap}, \mathrm{jet}}^{2} \equiv \frac{4 \pi \rho_{\mathrm{jet}} v_{\mathrm{p}, \mathrm{jet}}^{2}}{B_{\mathrm{p}, \mathrm{jet}}^{2}},
$$

and the (squared) jet internal total Alfvén Mach number,

$$
M_{\mathrm{A}, \mathrm{jet}} \equiv \frac{4 \pi \rho_{\mathrm{jet}} v_{\mathrm{p}, \mathrm{jet}}^{2}}{B_{\mathrm{p}, \mathrm{jet}}^{2}+B_{\phi, \mathrm{jet}}^{2}} .
$$

With the (squared) jet internal sound speed $c_{\mathrm{s}, \mathrm{jet}}^{2}=$ $\gamma P_{\text {jet }} / \rho_{\text {jet }}$ and Mach number $M_{\mathrm{S}, \text { jet }} \equiv v_{\mathrm{p}, \mathrm{jet}} / c_{\mathrm{s}, \mathrm{jet}}$ the jet internal fast magnetosonic Mach number is

$$
M_{\mathrm{F}, \mathrm{jet}} \equiv\left(M_{\mathrm{A}, \mathrm{jet}}^{-2}+M_{\mathrm{S}, \mathrm{jet}}^{-2}\right)^{-1 / 2} .
$$

The external Mach number relates the poloidal jet velocity to the sound speed in the external medium, $M_{\mathrm{S} \text {,ext }} \equiv$ $v_{\mathrm{p}, \text { jet }} / c_{\mathrm{s}, \mathrm{ext}}$.

Note that the Mach numbers are defined in respect to the poloidal velocity, considering MHD waves in the jet propagation direction. Tables 2 , 4 summarizes the parameter setup for the 1.5D simulations, whereas Tab. 3 shows the parameter space for the $2.5 \mathrm{D}$ simulations.

The initial magnetic field $B_{\mathrm{z}}$-component is constant over the whole computational domain. We run simulations for which the toroidal field component $B_{\phi}$ is injected into the ambient gas by the jet and others where a shock propagates along an already existing helical field structure. For the jet toroidal field we have applied a linearly increasing radial profile and a sine-profile. While the linear profile is force-free within the jet, we find the the sharp cut-off at the jet boundary leads to current sheets which disturb the flow ab initio and could not be accepted. We therefore discuss the simulations applying the (non force-free) sine profile only.

\section{RESULTS AND DISCUSSION}

We first discuss simulations resulting from a $1.5 \mathrm{D}$ setup. These can be considered as toy simulations of axisymmetric shocks in a helical field and test cases of the model. We then continue with $2.5 \mathrm{D}$ axisymmetric simulations of jet propagation with parameter ranges more comparable to observational values.

\subsection{Jet shock propagation in $1.5 \mathrm{D}$}

Quite some literature exists on one-dimensional MHD shock simulations, as this setup provides one of the standard tests for MHD codes. In particular we refer to Uchida et al. (1992); Ryu \& Jones (1995). We decided to repeat and extend a few of these 1.5D simulations for the following reasons. Firstly, they serve us as test case for our code for the $1.5 \mathrm{D}$ setup in cylindrical coordinates as we can directly compare them to simulations in the literature in Cartesian coordinates which are widely used also for analytic considerations. Secondly, this simple setup will most clearly demonstrate the essential mechanism leading to a toroidal jet acceleration across a shock. Thirdly, it allows us easily to explore the typical parameter space for protostellar jets (see Tab. 2). In the majority of our simulations the jet is injected as nonrotating. This simplification is made in order to demonstrate the acceleration mechanism by the shock, and is in general only valid for a certain iso-rotation parameter $\Omega_{\mathrm{F}}=\left(v_{\mathrm{p}} B_{\phi}\right) /\left(B_{\mathrm{p}} r\right)$.

We have performed several test simulations, of which two are shown in the appendix. That is simulation RJ11 repeating the simulation by Ryu \& Jones (1995) (see their Fig. 2a), done as well in Cartesian coordinates (Fig. 14). Simulation RJ09 is an extension of this parameter setup using however cylindrical coordinates (Fig. 15) and considering only one perpendicular vector component (no radial magnetic field, no radial motion).

Our first science example is a shock moving along a helical magnetic field (run RJ17), similar to the setup of Ryu \& Jones (1995). This is comparable to a jet internal shock propagating within the jet channel, caused e.g. by a sudden increase of jet injection velocity. Figure 1 shows the dynamical state after two dynamical time scales. The typical shock structure is visible, consisting of four shocks which propagate back resp. forth in respect to the contact discontinuity. The hydrodynamical state of the different shock regions varies, each satisfying the Rankine-Hugoniot jump conditions. The toroidal velocity panel in Fig. 1 clearly shows how the material is set into rotation when crossing the shock fronts.

Across the shock the toroidal field becomes compressed, and the steeper field gradient implies a toroidal torque on the shocked material. The material downstream and upstream the shock rotates in opposite direction, seen also in axial profile of kinetic angular momentum. This is a consequence of a change of sign 
Table 2

Parameter of axisymmetric 1.5D simulations. The external density is $\rho_{\text {ext }}=1.0$ for all runs. The toroidal velocity is given before and after the shock front $v_{\phi, 1}, v_{\phi, 2}$. Remarks: UT05 follows the same parameter setup as UT04, but considers cooling. UT06 follows the same parameter setup as UT04, except for the negative rotation velocity. For comparison with Figs. 1 [2 note the PLUTO magnetic field normalization $B \rightarrow B / \sqrt{4 \pi}$.

\begin{tabular}{cccccccccccccc}
\hline ID & $\rho_{\text {jet }}$ & $v_{\mathrm{p}, \text { jet }}$ & $v_{\phi, \text { jet }}$ & $P_{\text {jet }}$ & $P_{\text {ext }}$ & $B_{z}$ & $B_{\phi, \text { jet }}$ & $B_{\phi, \text { ext }}$ & $M_{\mathrm{Ap}, \text { jet }}$ & $M_{\mathrm{A}, \text { jet }}$ & $M_{\mathrm{F}, \text { jet }}$ & $M_{\mathrm{S}, \text { ext }}$ & $v_{\phi, 1}, v_{\phi, 2}$ \\
\hline RJ03 & 2.5 & 1.0 & 0.0 & 1.53 & 0.27 & 0.11 & 1.70 & 0.0 & 50.0 & 3.5 & 0.88 & 1.5 & $0.0 /-0.163$ \\
RJ04 & 2.4 & 1.0 & 0.0 & 0.32 & 0.27 & 0.11 & 1.56 & 0.0 & 50.0 & 3.5 & 1.82 & 1.5 & $0.005 /-0.235$ \\
RJ06 & 2.4 & 1.0 & 0.0 & 0.026 & 0.27 & 0.18 & 1.00 & 0.0 & 30.0 & 5.5 & 4.42 & 1.5 & $0.017 /-0.22$ \\
RJ17 & 1.08 & 1.2 & 0.0 & 0.95 & 1.0 & 2.0 & 2.0 & 2.0 & 2.21 & 1.56 & 0.84 & 0.93 & $+0.091 /-0.088$ \\
RJ18 & 1.08 & 1.2 & 0.0 & 0.95 & 1.0 & 2.0 & 2.0 & 0.0 & 2.21 & 1.56 & 0.84 & 0.93 & $+0.084 /-0.31$ \\
RJ19 & 1.28 & 1.5 & 0.0 & 1.15 & 1.0 & 2.0 & 2.0 & 0.0 & 3.01 & 2.13 & 1.06 & 1.16 & $+0.078 /-0.29$ \\
RJ21 & 1.28 & 1.2 & -0.2 & 0.95 & 1.0 & 2.0 & 2.0 & 0.0 & 2.41 & 1.70 & 1.10 & 0.93 & $-0.12 /-0.41$ \\
RJ22 & 1.28 & 1.2 & 0.0 & 0.95 & 1.0 & 0.5 & 2.0 & 0.0 & 9.63 & 2.34 & 0.98 & 0.93 & $+0.019 /-0.33$ \\
RJ23 & 1.28 & 1.2 & 0.0 & 0.95 & 1.0 & 2.0 & 0.5 & 0.0 & 2.41 & 2.34 & 0.98 & 0.93 & $+0.029 /-0.078$ \\
UT01 & 1.0 & 4.0 & 0.0 & 0.1 & 0.01 & 4.0 & 2.4 & 2.4 & 3.55 & 3.04 & 2.90 & 31.0 & $+0.7 /-0.7$ \\
UT02 & 1.0 & 4.0 & 0.0 & 0.1 & 0.01 & 4.0 & 2.4 & 0.0 & 3.55 & 3.04 & 2.90 & 31.0 & $+0.7 /-0.4$ \\
UT03 & 1.0 & 4.0 & 0.0 & 0.1 & 0.01 & 1.0 & 2.4 & 0.0 & 14.2 & 5.44 & 4.76 & 31.0 & $+0.14 /-0.54$ \\
UT04 & 1.0 & 4.0 & 0.1 & 0.1 & 0.01 & 1.0 & 2.4 & 0.0 & 14.2 & 5.44 & 4.76 & 31.0 & $+0.24 /-0.48$ \\
UT05 & 1.0 & 4.0 & 0.1 & 0.1 & 0.01 & 1.0 & 2.4 & 0.0 & 14.2 & 5.44 & 4.76 & 31.0 & $+0.25 /-0.46$ \\
UT06 & 1.0 & 4.0 & -0.1 & 0.1 & 0.01 & 1.0 & 2.4 & 0.0 & 14.2 & 5.44 & 4.76 & 31.0 & $+0.043 /-0.58$ \\
\hline
\end{tabular}

in the toroidal Lorentz force $F_{\phi} \sim B_{z} \partial B_{\phi} / \partial z$ across the shock. With increasing time, the shock evolution demonstrates the continuous conversion of magnetic angular momentum flux into kinetic angular momentum flux (not shown here, but see Figs. 1213 below). The the domain-integrated kinetic angular momentum increases in time. Due to the downstream boundary condition for the toroidal magnetic field, the net amount of kinetic angular momentum along the flow remains zero: Implicitly, the outflow conditions for angular momentum is kept the same as the inflow condition.

Our next example RJ18 applies similar flow parameters, but corresponds to a model setup where a jet is injected into an ambient medium threaded by an axial magnetic field only (the ambient toroidal magnetic field is set to zero). Figure 2 shows the dynamical state at the same time as for RJ17. The difference comes from the magnetic angular momentum flux which now decreases along the flow, while being transferred to kinetic angular momentum. For the parameters applied the toroidal velocities obtained are about $25 \%$ of the jet velocity, and much higher than the observed values.

Simulations RJ17 and RJ18 exhibit a similar hydrodynamic shock structure (considering $v_{\text {jet }}, \rho, P$ ). Because of the stronger toroidal field coiling, the MHD torque and, thus, the resulting toroidal velocities are increases by a factor of three.

Figure 3 shows simulation RJ21 in which a jet rotating with $v_{\phi \text {,jet }}=-0.2$ is injected into the ambient medium. By choice, the injected toroidal field direction is antialigned with the toroidal velocity. The shock structure of RJ21 is very similar to RJ18 which has otherwise the same hydrodynamic and magnetic parameters. Comparing RJ18 to RJ21, the resulting maximum jet rotational velocity is enhanced from $v_{\phi}=-0.31$ to $v_{\phi}=-0.41$, while the rotational velocity jump across the receding shock is the same in both cases, $\Delta v_{\phi}=0.74 . v_{\phi}=-0.41$, but also from $v_{\phi}=-0.31$ to $v_{\phi}=-0.41$. Figure 3 also shows the hydrodynamic and the magnetic angular momentum contribution to the total angular momentum budget (see Eq. 1, but note that we measure the a.m. at unity radius $r=1$ ). For each of the four shocks the total specific angular momentum is conserved in the shock frame satisfying the Rankine-Hugoniot conditions. It can be clearly seen that the magnetic angular momentum decreases across the shock as the kinetic angular momentum contribution increases.

It is also interesting to compare simulations RJ22 and RJ23 (see Figs. 4, 5). Both start off with the same initial magnetic angular momentum budget and Poynting flux $\sim B_{\mathrm{p}} B_{\phi}$, however, RJ22 has $B_{\phi}=4 B_{\mathrm{p}}$, while for RJ23 it is $B_{\mathrm{p}}=4 B_{\phi}$. From this, one may expect also a similar toroidal magnetic torque and, thus, a similar toroidal velocity, in particular since a similar compression rate in the hydrodynamic parameters and also in $B_{\phi}$ applies. However, we find that the resulting maximum toroidal velocities substantially change from 0.19 to 0.29 for the receding shock, or from -0.33 to -0.078 for the preceding shock and similarly for the kinetic angular momentum (see Fig. 4). The reason is that the stronger poloidal field in RJ22 broadens the shock structure and thus distributes the kinetic angular momentum over a wider spatial area. This is a typical property of C-shocks. Note, however, that the total, volume-integrated kinetic angular momentum in the shocked region is the same since the volume of the shocked gas differs (the shock widens from $\Delta r=1.5$ to 3.5 for the preceding shock).

We have also performed a comparison simulation with applying a simple power-law cooling function. Our simulations indicate a minor effect of cooling on jet rotation. The shock width decreases slightly, as the gas pressure decreases by cooling (this is a $10 \%$-effect for the chosen cooling function). The gas density and toroidal magnetic field strength increase resulting in a slightly increased maximum toroidal velocity. These preliminary results need to be further investigated in a future paper.

In summary, our 1.5D simulations clearly show how a jet flow in a helical magnetic field is forced into rotation, when the toroidal field component is compressed across the shock, thus exerting a Lorentz force on the 

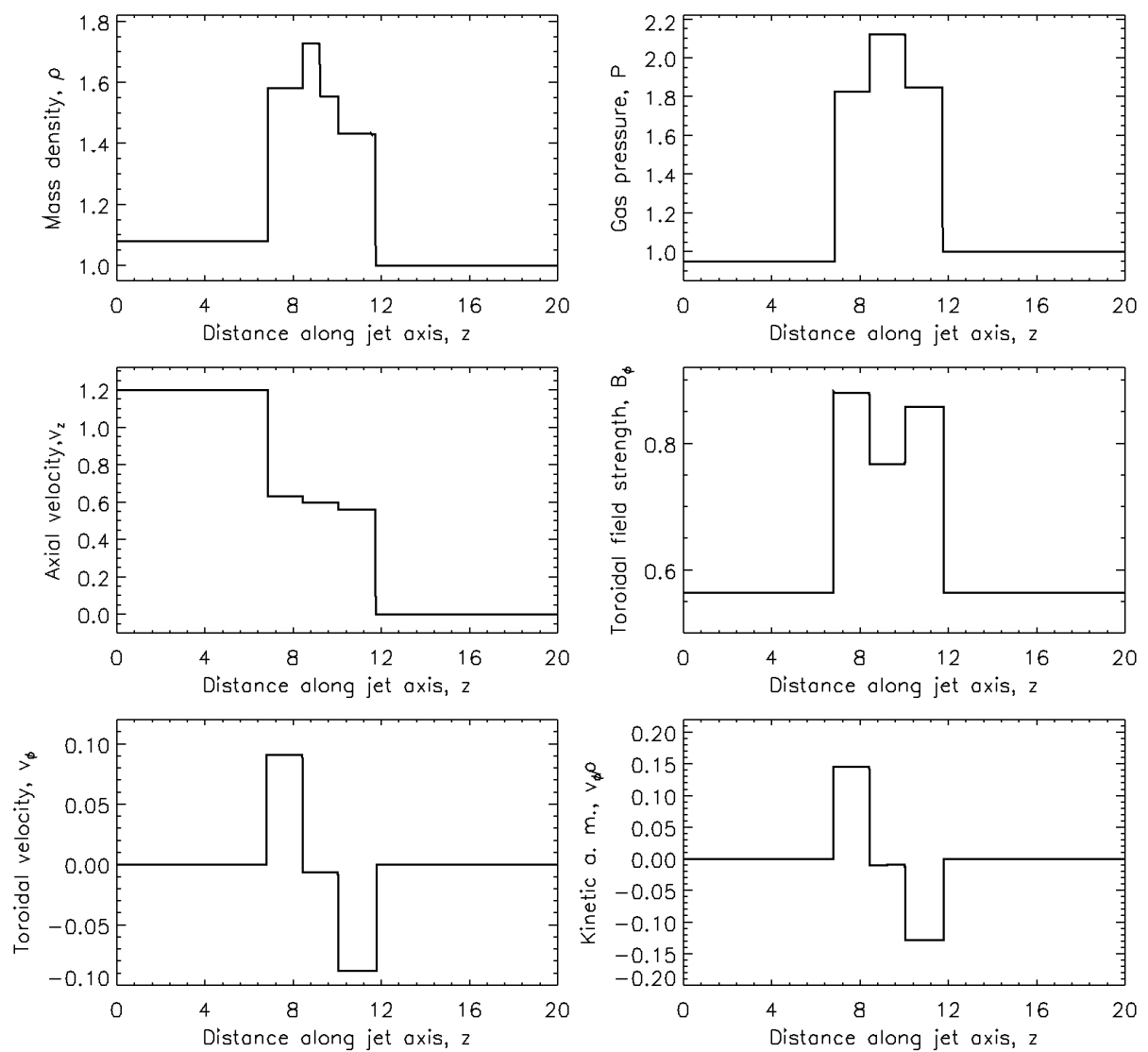

Figure 1. Axisymmetric $1.5 \mathrm{D}$ shock simulation RJ17 in cylindrical coordinates. Shock evolution at dynamical time $t=2$ with a grid resolution of 1.000 cells / unit length. Shown is density; gas pressure; axial, and toroidal velocities; axial, and toroidal magnetic field strengths, and the kinetic angular momentum (at $r=1$ ) (from top left to bottom right).

jet material. Typically, jet rotation velocities are about $3-10 \%$ of the jet axial velocity for Alfvén Mach numbers $M_{\mathrm{Ap}, \mathrm{jet}} \simeq 2-3$ (see Tab. 2 ).

\subsection{Jet shock propagation in 2.5D}

We now discuss axisymmetric simulations taking into account all 3 vector components. This approach allows to investigate also the lateral expansion of the jet and its interaction with the ambient medium. We have run a parameter study, mainly considering different values for the jet magnetosonic Mach numbers and plasma beta (see Tab. 3).

The general boundary condition applied for the jet toroidal magnetic field follows a profile $B_{\phi}=$ $B_{\phi, \max }(\sin ((2.0 r-0.5)) \pi+1.0)$ for $r<1.0$, and vanishes for larger radii. This is not a force-free configuration, however, it avoids the strong current sheet usually launched by sawtooth-like profiles. The initial poloidal field distribution is constant and purely longitudinal. The radial profile of the jet injection velocity follows a cosine profile, $v_{\text {inj }}(r)=v_{\text {jet }} \cos (r \pi / 2)$ for $r<1$. We have also run simulations with a time-dependent injection velocity, $v_{\text {jet }}(t)=1.0+0.5 * \sin (0.3 t)$, thus $v_{\text {inj }}(t, r)=v_{\text {inj }}(r) *(1.0+0.5 * \sin (0.3 t))$, or, alternatively with a longer period $\sim(1.0+0.5 * \sin (0.1 t))$.

Simulation run R01 considers a high external Mach number $M_{\mathrm{s} \text {,ext }}=10$, a comparatively weak jet magnetic field corresponding to high magnetosonic Mach numbers, and a high plasma beta - thus, a jet rather close to the hydrodynamical limit. Figure 6 shows the time evolution of the toroidal velocity of this jet for the dynamical time steps $t=10,30,40$, and the corresponding toroidal magnetic field strength, axial velocity, and toroidal torque for time $t=30$. This images show the inner domain $r<5.0$ of the simulation box $r<7.0$.

The simulation shows the rotating material surrounding the inner jet beam in a cocoon-like structure. The injected jet material is non-rotating (by definition of the boundary condition). For run R01 the obtained rotational velocities are comparatively low $v_{\phi}<$ $10^{-4} v_{\text {jet}}$, resulting from the low magnetic field strength in spite of the high shock compression ratio in the nearhydrodynamical limit.

During the initial time steps the termination shock is rotating with positive sense, the bow shock with negative sense. The toroidal magnetic field compression is clearly seen in the termination shock, but also in surrounding cocoon. The cocoon toroidal field is enhanced partly by the radial expansion of the shocked gas, partly by winding up the poloidal field by the rotating jet material.

The map of the toroidal torque demonstrates the interrelation between Lorentz force and rotation. The jet head is rotating in positive direction $(t=40$, grey blackish colors) with the toroidal torque in the same direction (white colors). The rotation velocity measured at a certain time is, however, resulting from the time-integrated torque on a parcel of material along its path.

Simulation run R12 considers a high (external) Mach number jet with weak magnetic field, similar to R01, but representing a less extreme case (see Fig. 7). The toroidal 

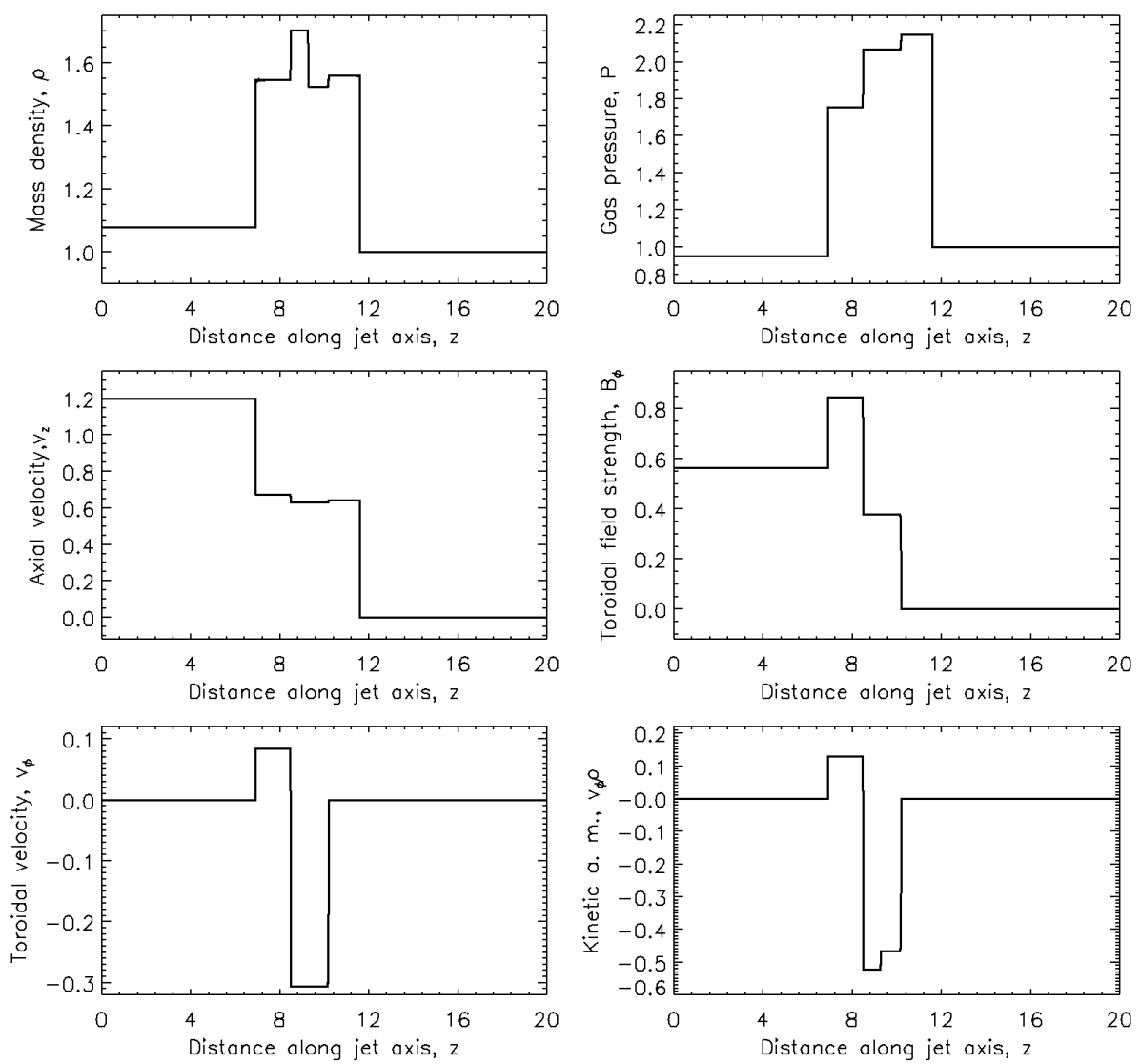

Figure 2. Axisymmetric $1.5 \mathrm{D}$ simulation RJ18 in cylindrical coordinates. Shock evolution at dynamical time $t=2$ with a grid resolution of 1.000 cells / unit length. Shown is density; gas pressure; axial, and toroidal velocities; axial, and toroidal magnetic field strengths, and the kinetic angular momentum (at $r=1$ ) (from top left to bottom right). The toroidal magnetic field in the ambient medium is vanishing (compare to simulation RJ17 in Fig. 1).

velocities induced range from -0.07 to 0.033 corresponding to $1-3 \%$. This is in the range of the observationally indicated velocities. Also the jet dynamical parameters, $M_{\mathrm{A}, \mathrm{jet}}=30, M_{\mathrm{S}, \mathrm{ext}}=5$, are observationally indicated. Simulation run R13 considers the same parameters except a lower plasma- $\beta$, thus a stronger poloidal magnetic field.

For jets with lower Mach numbers (e.g. R09, R10) even higher toroidal velocities can be produced. The main contribution to the rotating material is from entrained (and shocked) ambient gas.

Simulation R16 runs with the same parameter setup as R12, but in difference applies a time-dependent injection speed, $v_{\text {jet }}(t)=v_{\text {jet }, 0}(1.0+0.5 \sin (0.3 t))$. This leads to subsequent internal shocks along the jet, which due to $B_{\phi}$-compression also force the jet material in rotation (see Fig. 8, 9). In addition, each of the subsequent shock fronts further entrains ambient material. Accompanying shocks set this material into rotation as well. For this setup, it might be interesting to estimate the astrophysical parameters. Assuming a jet radius of $20 \mathrm{AU}$, the separation between the generated knots would correspond to about $600 \mathrm{AU}$ (one grid length). The knot pattern separation would then correspond to a time scale of 15 years, estimated from the 50 time units the knot pattern needs to traverse the $600 \mathrm{AU}$ grid (one time unit $\Delta t=1$ corresponds to about $10^{7} \mathrm{~s}$ assuming a jet mean velocity of $300 \mathrm{~km} / \mathrm{s}$ ). This time scale is of course put in by hand, but is not very different from observationally derived numbers.

Simulation run R19 with lower Mach / Alfvén Mach numbers compared to R16 has a slightly higher frequency in the injection speed variation. Together this results in more internal substructure, seen e.g. in the toroidal velocity distribution (see Fig. 10), but also in the other dynamical variables (not shown). (and density, 6 not shown).

\subsection{Oblique reflection shocks}

Jets with low (internal) Mach numbers develop standing oblique relection shocks internal to the jet flow which do not further move as jet and bow shock propagate. As an example Fig. 11 shows the dynamical state of simulation run R12 at an intermediate time step $t=50$. The reflection shocks are most clearly seen in the density distribution and the radial velocity distribution. The change in propagation speed along the jet axis is about $15 \%$ across each shock region.

Along the jet flow the toroidal Lorentz force is predominantly negative leading to an according rotation of the jet material. The shock structure consists of a thin layer with positively directed toroidal Lorentz force followed (direction downstream) by a extended region of positively directed toroidal Lorentz force. The latter force is dominating leading to an overall negative rotation of the jet beam.

Comparing the toroidal Lorentz force and toroidal velocity distribution in Fig. 11 gives interesting insight on 
Table 3

Parameter study of axisymmetric $2.5 \mathrm{D}$ jet simulations. The ambient density is $\rho_{\text {ext }}=1.0$ for all simulations. The minimum and maximum values for the toroidal velocity $\left\langle v_{\phi, j e t,(\min , \max )}\right\rangle$ are derived from a histogram statistics, i.e. indicating the maxima for the bulk toroidal motion (there is always a tail in the toroidal velocity distribution of even higher velocities). The toroidal velocity maxima are estimates when the jet flow has penetrated most of the computational domain, but its bow shock has not yet left the grid.

\begin{tabular}{|c|c|c|c|c|c|c|c|c|c|c|}
\hline ID & $\rho_{\text {jet }}$ & $v_{\text {jet }}$ & $P_{\text {jet }}$ & $P_{\text {ext }}$ & $M_{\mathrm{Ap}, \text { jet }}$ & $M_{\mathrm{A}, \text { jet }}$ & $M_{\mathrm{S}, \text { ext }}$ & $\beta$ & $<v_{\phi, \text { jet },(\min , \max )}>$ & Remarks \\
\hline R01 & 5.5 & 1.0 & 0.006 & 0.0015 & 2000.0 & 300.0 & 10.0 & 20.1 & $-0.00014,0.000065$ & \\
\hline R02 & 0.5 & 1.0 & 0.002 & 0.006 & 200.0 & 30.0 & 10.0 & 10.1 & $-0.0023,0.04$ & \\
\hline R03 & 1.0 & 1.0 & 0.015 & 0.006 & 200.0 & 30.0 & 10.0 & 10.1 & $-0.028,0.023$ & \\
\hline R04 & 1.0 & 1.0 & 0.012 & 0.024 & 200.0 & 30.0 & 10.0 & 5.0 & $-0.022,0.025$ & \\
\hline R05 & 1.0 & 1.0 & 0.9 & 0.15 & 20.0 & 3.0 & 10.0 & 2.1 & $-0.42,0.25$ & \\
\hline R06 & 1.0 & 1.0 & 0.9 & 0.15 & 50.0 & 3.0 & 10.0 & 1.0 & $-0.67,0.40$ & \\
\hline R07 & 1.0 & 1.0 & 0.9 & 0.15 & 50.0 & 3.0 & 10.0 & 2.0 & $-0.55,0.40$ & \\
\hline R08 & 1.0 & 1.0 & 0.5 & 0.15 & 50.0 & 3.0 & 0.5 & 2.0 & $-0.22,0.15$ & \\
\hline R09 & 1.0 & 1.0 & 0.5 & 0.15 & 50.0 & 3.0 & 0.5 & 2.0 & $-0.15,0.07$ & \\
\hline $\mathrm{R} 11$ & 1.0 & 1.0 & 0.1 & 0.15 & 20.0 & 3.0 & 2.0 & 1.1 & $-0.18,0.05$ & \\
\hline $\mathrm{R} 12$ & 1.0 & 1.0 & 0.001 & 0.024 & 200.0 & 30.0 & 5.0 & 1.1 & $-0.0023,0.015$ & \\
\hline $\mathrm{R} 13$ & 1.0 & 1.0 & 0.0001 & 0.024 & 200.0 & 30.0 & 5.0 & 0.1 & $-0.0031,0.010$ & \\
\hline $\mathrm{R} 15$ & 1.0 & 1.0 & 0.01 & 0.067 & 50.0 & 10.0 & 3.0 & 1.1 & $-0.03,0.05$ & $\Delta v_{\mathrm{inj}} \sim \frac{1}{2} \sin (0.1 t)$ \\
\hline $\mathrm{R} 16$ & 1.0 & 1.0 & 0.001 & 0.024 & 50.0 & 10.0 & 3.0 & 1.1 & $-0.007,0.007$ & $\Delta v_{\mathrm{inj}} \sim \frac{1}{2} \sin (0.3 t)$ \\
\hline $\mathrm{R} 17$ & 1.0 & 3.0 & 0.01 & 0.22 & 200.0 & 30.0 & 5.0 & 1.1 & $-0.0035,0.013$ & $\Delta v_{\mathrm{inj}} \sim \frac{1}{2}(\sin 0.5 t)^{3}$ \\
\hline R19 & 1.0 & 1.0 & 0.05 & 0.15 & 50.0 & 5.0 & 2.0 & 1.1 & $-0.035,0.05$ & $\Delta v_{\mathrm{inj}} \sim \frac{1}{2}(\sin 0.2 t)^{3}$ \\
\hline
\end{tabular}

the acceleration process. The positive forces are strongly localized across the relection shocks. However, we don't see there effect in a positive jet rotation with in the jet beam. In comparison, the negative forces are distributed over a wide range between the internal shocks. As a result the (negative) rotation velocity of the jet beam increases along the propagation direction. The increase is step-wise with each of the inter-shock region adding to the overall rotation (see the step-wise increase of dark color along the jet flow in the upper right Fig. 11).

Along the jet beam the negative Lorentz force wins and the overall jet rotation is negative. The situation is somewhat different for the material which is entrained along the jet flow. Here the negative Lorentz force is more strongly localized and the positive force wins resulting in an overall positive rotation of the ambient (entrained) flow.

This picture of a gradual increase of rotation by cumulative action of Lorentz forces along the path of the material is also supported by the small rotation feature visible near the jet head. A closer look shows that the respective Lorentz force and velocity patterns are slightly shifted. This tells us the jet $\phi$-acceleration is not, say in situ, but that what we observe in rotation is the result of previously acting Lorentz forces. The same trend can be seen in the small scale toroidal velocity structures around the jet in the positive velocities where observe a similar shift between the positive Lorentz force and the positive toroidal velocity.

\subsection{Time evolution of angular momentum}

The magnetic field structure resulting from our rather basic and general simulation setup evolves into a complex helical field distribution implying a similarly complex electric current system. With the propagation of the outflow and the evolving jet-internal dynamics the toroidal and poloidal field components change direction resulting in a similar variation of the torques along and across the jet. This is demonstrated exemplarily in Fig. 6, where we show the distribution of the specific toroidal Lorentz forces, resp. the acceleration in toroidal direction. Comparison of positive (right) and negative (left) Lorentz forces, highlights the filamentary structure of the force distribution. The structural details clearly depend on the specifics of the simulation setup and deserve a closer look by a future investigation. Observational indications for such a filamentary structure may be obtained from radial velocity profiles across the jet if the spatial resolution will be sufficient.

The time-evolution shown in Fig. 6 is typical for the early jet evolution - the jet is penetrating the ambient medium and thereby changes the dynamical state compared to its initial injection profile. However, jet material and ambient gas are coupled by the magnetic field which is anchored into both components. The differential rotation between jet and ambient gas results in shear and torques. In later evolutionary stages when the jet has bored its funnel through the ambient gas, the differential rotation decreases, and the twist in the field structure is reduced. Jet injections of different velocity will then lead to internal shocks (see simulation R16, Fig. 8, 9).

Figure 12 reveals the time sequence of the angular momentum evolution as domain-integrated value for sim- 

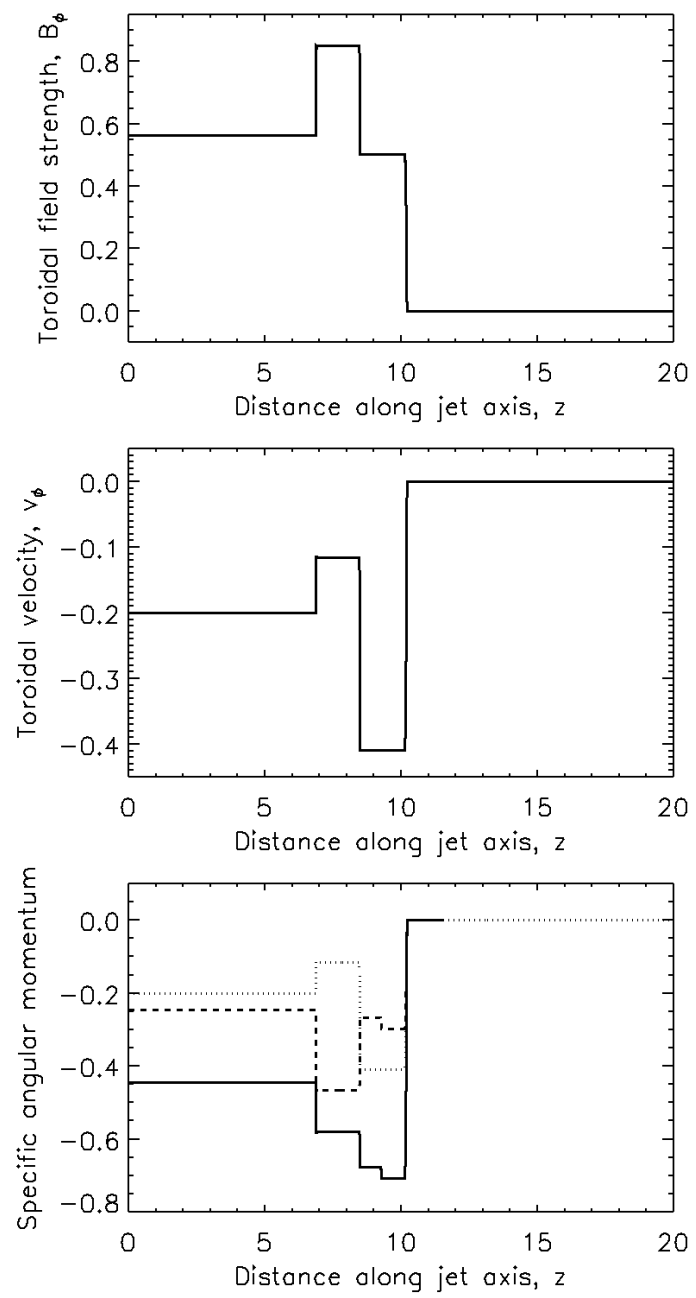

Figure 3. Axisymmetric 1.5D simulation of a propagating MHD shock. Simulation RJ21 considers a rotating jet injected into an ambient medium with $v_{\phi \text {,jet }}=-0.2$, but otherwise the same parameters as RJ18 (see Fig.22). Shown is the shock evolution at dynamical time $t=2$ with a grid resolution of 1.000 cells / unit length. The bottom panel shows the specific total angular momentum $\rho v_{\phi}$ (solid), with the magnetic (dashed), and hydrodynamic (dotted) contribution.

ulation R01. Shown are the different contributions to the total angular momentum budget (i.e. not their specific value). Since this is an density-weighted value, it is observationally more relevant than the specific value (simply speaking, more gas will radiate more). The observed jet rotation will not only depend on the rotation speed alone, but also on how much material is in fact rotating with such speed. The example of Fig. 12 shows at time $t=20$ the kinetic angular momentum on average more positive than negative. This suggests that one may observe on average a positive rotation of the jet although the negative velocity distribution is broader (see Fig. 6).

At dynamical time $t=45$ the jet bow shock leaves the computational domain. The jet material set into rotation by the processes we have discussed above will propagate out of the computational domain. Since simulation R01 has a time-independent injection boundary condition, no new shocks will be initiated which could enforce rotation and the overall jet rotation will fade away - as indicated by Fig. 6 .
As for simulation R01 we show in Fig. 13 the time evolution for the different contributions to the angular momentum budget for simulation R12. In difference to R01 the amount of angular momentum is constantly growing. This is due to the presence of the steady reflection shock which continue to convert magnetic angular momentum to kinetic angular momentum, even when the bow shock has left the computational domain.

\section{CONCLUSIONS}

We have performed axisymmetric MHD simulations of jet propagation into an ambient gas applying the PLUTO code. The goal of our study was to investigate how the gas flow in jets and outflows is set in rotation by Lorentz forces arising MHD shocks.

The process we discuss relies on a helical magnetic field structure which carries magnetic angular momentum from the jet base to the asymptotic jet. This could be converted into kinetic angular momentum by action of a toroidal torque which is induced by shock compression of the toroidal field. To demonstrate the applicability of the principal mechanism, for simplicity we have injected a non-rotating jet material into the ambient gas for most cases.

Our simulations show that the proposed mechanism is indeed feasible, and accelerates the jet in toroidal direction by Lorentz forces which are induced by a shock compressed toroidal magnetic field. Depending on the magnetohydrodynamic parameters of the jet flow, the resulting rotational velocities are in the range of $0.1 \%-1 \%$ of the jet propagation speed.

Jets with a high external Mach number gain the highest rotation speed. This is because the mechanism works most efficient in the termination shock.

The resulting velocity field is highly complex resulting in a filamentary structure in the toroidal torque and toroidal velocity distribution. The material which is set in rotation across the terminal shock, flows back respective to the propagating jet and forms a cocoon with a highly tangled rotation pattern.

Interestingly, of jets with the same initial magnetic angular momentum budget (i.e. same $\sim B_{\phi} B_{z}$ ), those with a low poloidal field (low magnetic flux) experience a stronger toroidal torque, and, thus gain higher rotational speed. This is a consequence of the higher compression rates due to the weaker poloidal field (more J-type than C-type shock).

For the jets with steady injection, the toroidal velocity field decays as soon as the bow shock has left the computational domain. On the long term the injected gas flows along the channel bored by the jet during previous time steps and only weakly interacts with the ambient medium. For jets with time-dependent injection, further shocks are generated at a constant rate maintaining the jet rotation.

Jets with low internal Mach number develop standing oblique reflection shocks which constantly (but stepwise) accelerate the jet material in toroidal direction.

In summary, by demonstrating the feasibility of toroidal jet acceleration by MHD shocks in a helical magnetic field, we propose the following implications.

i) Jets of young stars are typically seen in radiation of shocked gas. Thus we expect this material to be affected by toroidal torques along its path from the jet launch- 

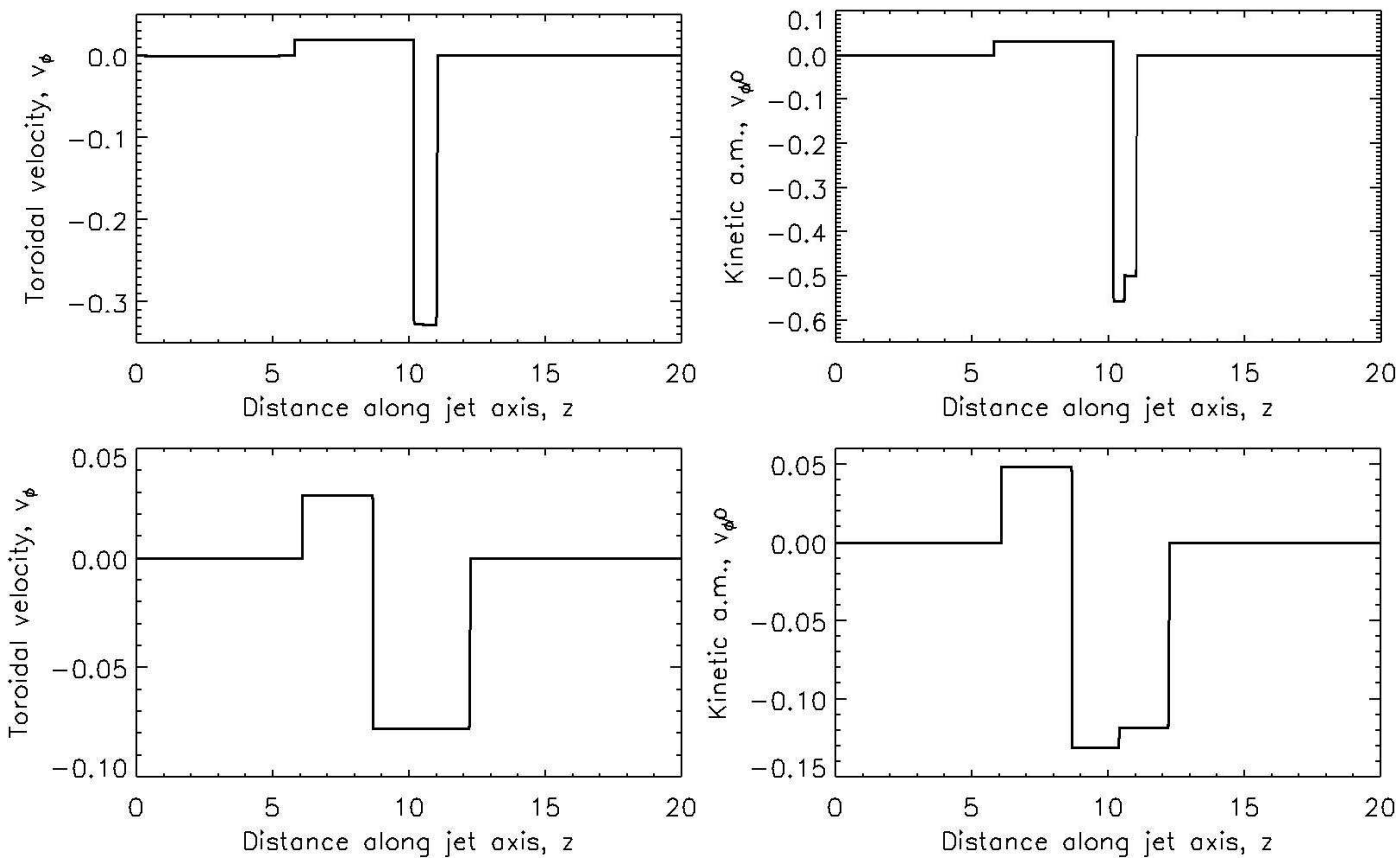

Figure 4. Axisymmetric 1.5D simulations RJ22 (top) and RJ23 (bottom) considering jets with same magnetic angular momentum $\sim B_{\phi} B_{z}$ (and Poynting flux), but different strength of the field components, $B_{\phi}=4 B_{z}$ (RJ22), $B_{z}=4 B_{\phi}$ (RJ23), respectively (with otherwise the same parameters). Shown is the toroidal velocity (left), and kinetic angular momentum (right) at dynamical time $t=4$.
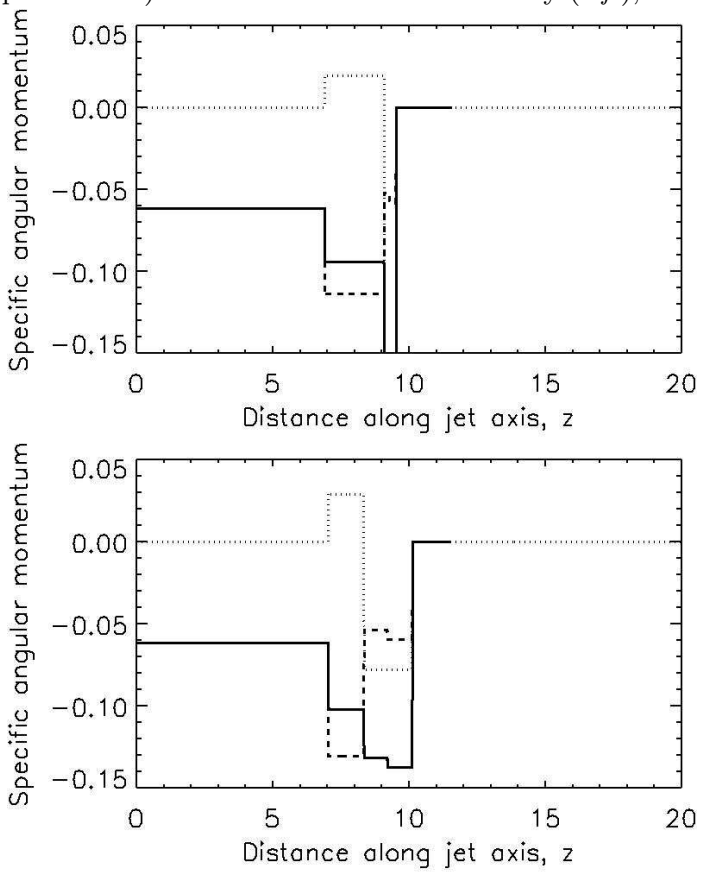

Figure 5. Axisymmetric 1.5D simulations RJ22 (top) and RJ23 (bottom) considering jets with the same magnetic angular momentum $\sim B_{\phi} B_{z}$ (and Poynting flux), but different strength of the field components, $B_{\phi}=4 B_{z}$ (RJ22), $B_{z}=4 B_{\phi}$ (RJ23), respectively (with otherwise the same parameters). Shown is the specific total angular momentum (solid), with the magnetic (dashed), and hydrodynamic (dotted) contribution.

ing region close to the star up to asymptotic regime of a collimated jet. To extrapolate from observed angular velocities far from the source to the angular momentum of the jet material close to its origin is therefore questionable.

ii) Jet rotation, as observationally indicated in jets from young stars is feasible. Jets emerge from rotating disks and are ejected with kinetic angular momentum. In addition to this, MHD shocks could additionally accelerate the jet material by further converting magnetic to kinetic angular momentum.

iii) Similar processes can be expected from relativistic stellar jets and extragalactic jets, leading to a rapid rotation in these jets.

The results presented in this paper are derived from kinetic / dynamical modeling. Preliminary simulations considering cooling indicate not major effect, although we see that cooling results in a somewhat stronger shock compression and therefore slightly higher maximum rotation speeds. Detailed comparison with observations would require also modeling of radiation processes (radiative MHD), or post-processing of emission maps, which is beyond the scope of this paper.

I thank the Andrea Mignone and the PLUTO team for the possibility to use their code. I also acknowledge many interesting and fruitful discussions with Bhargav Vaidya and Oliver Porth. My thanks go as well to an unknown referee who contributed a number of suggestions which clearly have improved the paper.

\section{APPENDIX}

\section{COMPARISON SIMULATIONS}

Here we show comparison simulations applying a similar set of parameters (see Tab. 4) as Ryu \& Jones (1995). Figure 14 shows simulation RJ11 which basically repeats the simulation shown in Ryu \& Jones (1995), Fig. 2a, for which we 

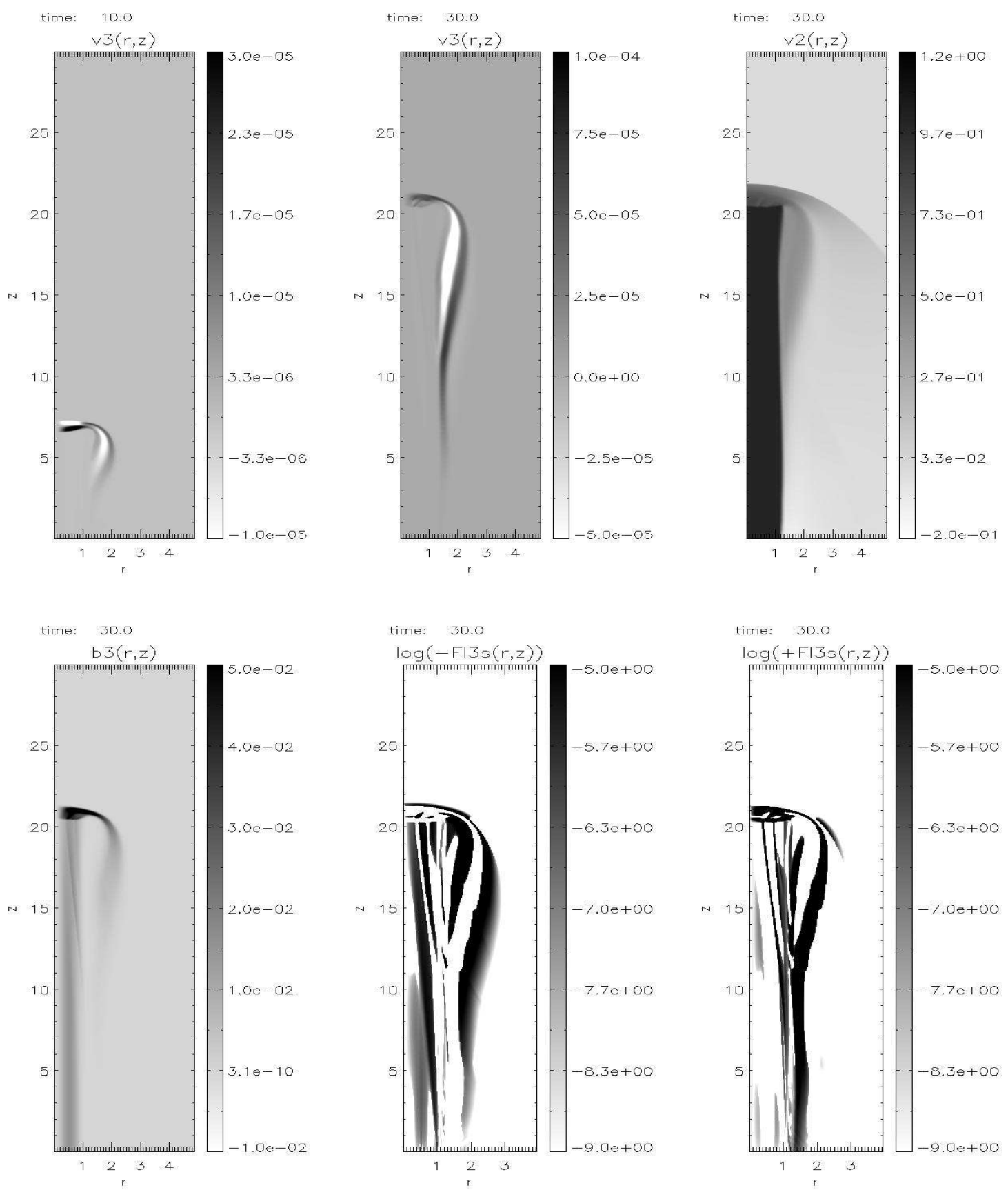

Figure 6. Axisymmetric 2.5D simulation run R01 considers a high (external) Mach number jet with weak magnetic field. Shown is (from top left to bottom right) the toroidal velocity at dynamical time $t=10,30$, and for the latter also the axial velocity $v_{z}$, the toroidal field strength $B_{\phi}$, and the negative and positive toroidal Lorentz force $r F_{\mathrm{L}, \phi}$ (on a log scale). Shown is the inner part $r<5.0$ of the whole grid of $(r<7.0, z<30.0)$. The inner, equidistant grid consists of $(100 \times 810)$ cells for $(r<1.5, z<30.0)$. Attached to that is a logarithmically scaled grid for $r>1.5$. The jet nozzle is located between $0<r<1.0$.

have applied Cartesian coordinates as well. Figure 15 shows simulation RJ09 with similar dynamical parameters, but run in a cylindrical coordinate system, which can be considered more appropriate to our general aim of investigating jet rotation. In the latter case we have considered only one perpendicular vector component. The initial setup with vanishing radial magnetic field and vanishing radial motion is very well conserved.

\section{REFERENCES}



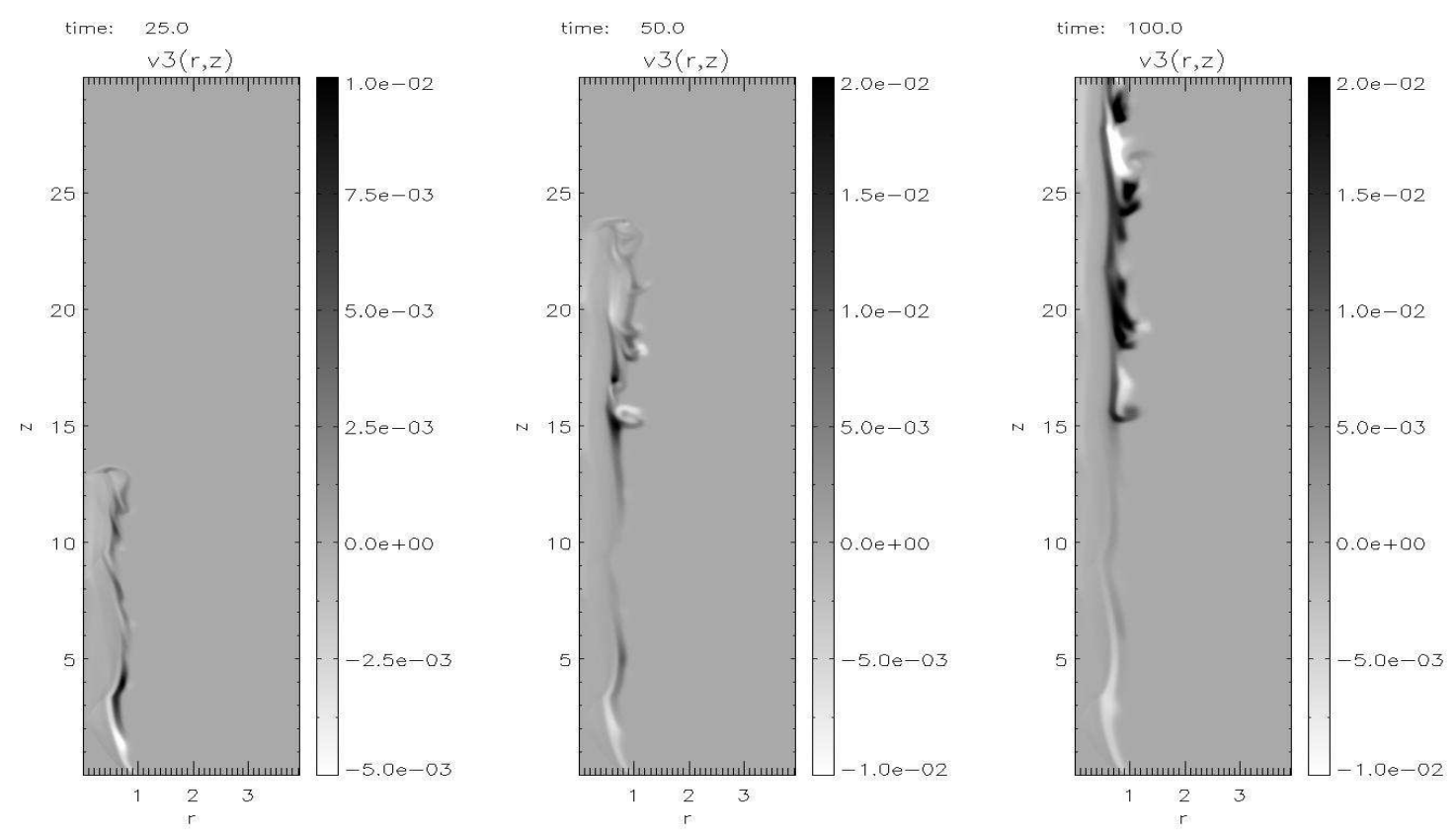

Figure 7. Axisymmetric simulation run R12 considers a high (external) Mach number jet with weak magnetic field, however, representing a less extreme case compared to run R01. Shown is toroidal velocity at time steps $t=25,50,100$ (from left to right) for a sub-grid of $r<4.0$ of the whole computational domain $(0.0<r<10.0,0.0<z<30.0)$.

Table 4

Parameter choice for the 1.5D simulations RJ09 (cylindrical coordinates) and RJ11 (Cartesian coordinates), which serve as test cases to be compared to Ryu \& Jones (1995). The ambient density is $\rho_{\text {ext }}=1.0$ For comparison with Figs. 15 14 note the magnetic field normalization $B \rightarrow B / \sqrt{4 \pi}$.

\begin{tabular}{ccccccccc}
\hline ID & $\rho_{\text {jet }}$ & $v_{\mathrm{p}, \text { jet }}$ & $v_{\phi, \text { jet }}$ & $P_{\text {jet }}$ & $P_{\text {ext }}$ & $B_{x}, B_{z}$ & $B_{y}, B_{r}$ & $B_{z}, B_{\phi}$ \\
\hline & & & & & & & & \\
RJ09 & 1.08 & 1.2 & 0.5 & 0.95 & 1.0 & $B_{z, \text { jet }}=B_{z, \text { ext }}=2.0$ & $B_{r, \text { jet }}=B_{r, \text { ext }}=0.0$ & $B_{\phi, \text { jet }}=B_{\phi, \text { ext }}=2.0$ \\
RJ11 & 1.08 & 1.2 & 0.5 & 0.95 & 1.0 & $B_{x, \text { jet }}=B_{x, \text { ext }}=2.0$ & $B_{y, \text { jet }}=3.6, B_{y, \text { ext }}=4.0$ & $B_{z, \text { jet }}=B_{z, \text { ext }}=2.0$
\end{tabular}

Bouvier, J., Alencar, S.H.P., Boutelier, T., Dougados, C., Balog, Z., Grankin, K., Hodgkin, S.T., Ibrahimov, M.A., Kun, M., Magakian, T.Y., Pinte, C. 2007, A\&A, 463, 1017

Cabrit, S., The accretion-ejection connexion in T Tauri stars: jet models vs. observations, in: J. Bouvier \& I. Appenzeller (eds.), Star-disk interaction in young stars, IAU Symposium 243, 2007, p.203

Camenzind, M. 1990, Magnetized disk-winds and the origin of bipolar outflows, in: G. Klare (ed.) Rev. Mod. Astron. 3, Springer, Heidelberg, p.234

Carrasco-González, C., Rodríguez, L.F., Anglada, G., Martí, J., Torrelles, J.M., Osorio, M. , 2010, Science, 330, 1209

Casse, F. \& Keppens, R. 2002, ApJ, 581, 988

Cecil, G., Wilson, A.S., Tully, R.B. 1992, ApJ 390, 365

Clarke, D.A., Norman, M.L., \& Burns, J.O. 1986, ApJ, 311, L63

Choi, M., Kang, M., Tatematsu, K. 2011, ApJ, 728, L34

Chrysostomou, A., Bacciotti, F., Nisini, B., Ray, T.P., Eislöffel, J., Davis, C.J., Takami, M. 2008, A\&A 482, 575

Coffey, D., Bacciotti, F., Woitas, J., Ray, T.P., Eislöffel, J. 2004, 604, 758

Correia, S., Zinnecker, H., Ridgway, S.T., McCaughrean, M.J. 2009, A\&A 505, 673

Davis, C.J., Berndsen, A., Smith, M.D., Chrysostomou, A., Hobson, J. 2000, MNRAS 314, 241

Dempsey, P. \& Rieger, F. 2009, IJMPD, 18, 1651

Fendt, C., Camenzind, M., \& Appl, S. 1995, A\&A, 300, 791

Fendt, C., \& Camenzind, M. 1996, A\&A, 313, 591

Fendt, C., \& Cemeljic, M. 2002, A\&A, 395, 1045

Fendt, C. 2006, ApJ, 651, 272

Fendt, C. 2009, ApJ, 692, 346

Ferreira, J., Pelletier, G., Appl, S. 2000, MNRAS, 312, 387

Frank, A., Lery, T., Gardiner, T.A., Jones, T.W., Ryu, D. 2000, ApJ, 540, 342

Gabuzda, D.C., Murray, E., Cronin, P. 2004 MNRAS, L351, 89

Herrnstein, J., Moran, J.M., Greenhill, L.J., Diamond, P.J., Miyoshi, M., Nakai, N., Inoue, M. 1997, ApJ, 475, L17

Kato, S.X., Kudoh, T., \& Shibata, K. 2002, ApJ, 565, 1035

Kössl, D., Müller, E. \& Hillebrandt, W. 1990, A\&A 229, 378

Krasnopolsky, R., Li, Z.-Y., \& Blandford, R. 1999, ApJ, 526, 631

Laing, R.A., Canvin, J.R., Bridle, A.H., Hardcastle, M.J. 2006, MNRAS, 372, 510 

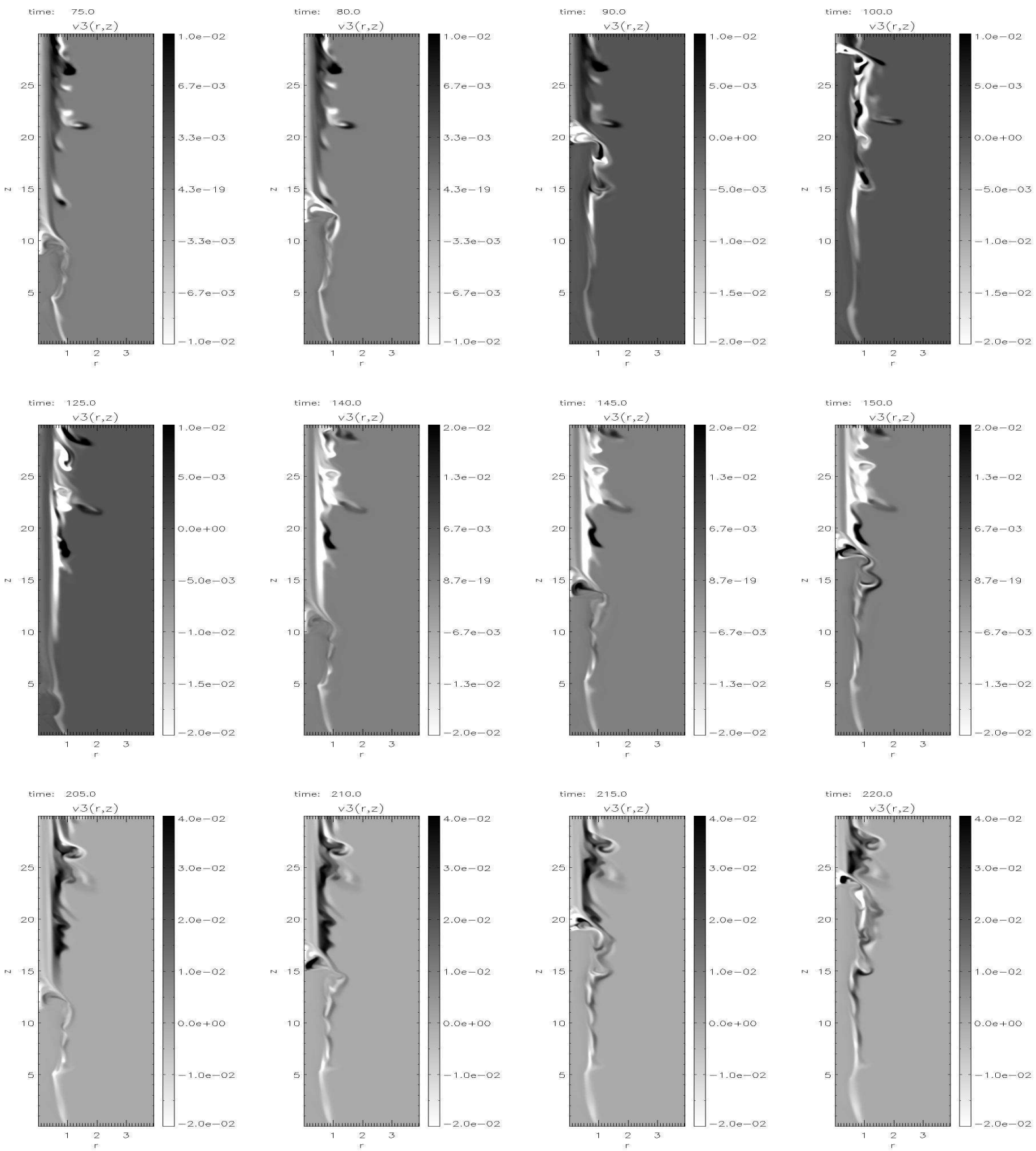

Figure 8. Axisymmetric simulation run R16 considers a high Mach number jet similar to R12, however with a time-dependent injection speed, thus generating internal shock waves moving along the jet. Shown is the toroidal velocity at dynamical time steps $t=75,80,90,100,125,140,145,150,205,210,215,220$. (from top left to bottom right) for a sub-grid of $r<4.0$ of the whole computational domain $(0.0<r<10.0,0.0<z<30.0)$. The jet nozzle is between $0<r<1.0$.

Launhardt, R., Pavlyuchenkov, Y., Gueth, F., Chen, X., Dutrey, A., Guilloteau, S., Henning, Th., Piétu, V., Schreyer, K., Semenov, D., 2009, A\&A, 494, 147

Lavalley-Fouquet, C., Sabrit, S., Dougadous, C. 2000, A\&A, 356, L41

Lee, C.-F., Ho, P.T.P., Palau, A., Hirano, N., Bourke, T.L., N., Shang, H., Zhang, Q. 2007, ApJ, 670, 1188

Lee, C.-F., Ho, P.T.P., Bourke, T.L., Hirano, N., Shang, H., Zhang, Q. 2008, ApJ, 685, 1026

Lee, C.-F., Hirano, N., Palau, A., Ho, P.T.P., Bourke, T.L., Zhang, Q., Shang, H. 2009, ApJ, 699, 1584

Lyutikov, M., Pariev, V.I., Gabuzda, D.C. 2005, MNRAS, 360, 869

Matt, S.P., Pudritz, R.E. 2008, ApJ, 678, 1109

Matt, S.P., Pinzon, G., de la Reza, R., Greene, T.P. 2010, ApJ, 714, 989

Meliani, Z., \& Keppens, R. 2007, A\&A 475, 785

Mignone, A., Bodo, G., Massaglia, S., Matsakos, T., Tesileanu, O., Zanni, C., Ferrari, A. 2007, ApJS, 170, 228

Moll, R. A\&A, 507, 1203

Movsessian, T.A., Magakian, T.Y., Bally, J., Smith, M.D., Moiseev, A.V., Dodonov, S.N. 2007, A\&A, 470, 605 

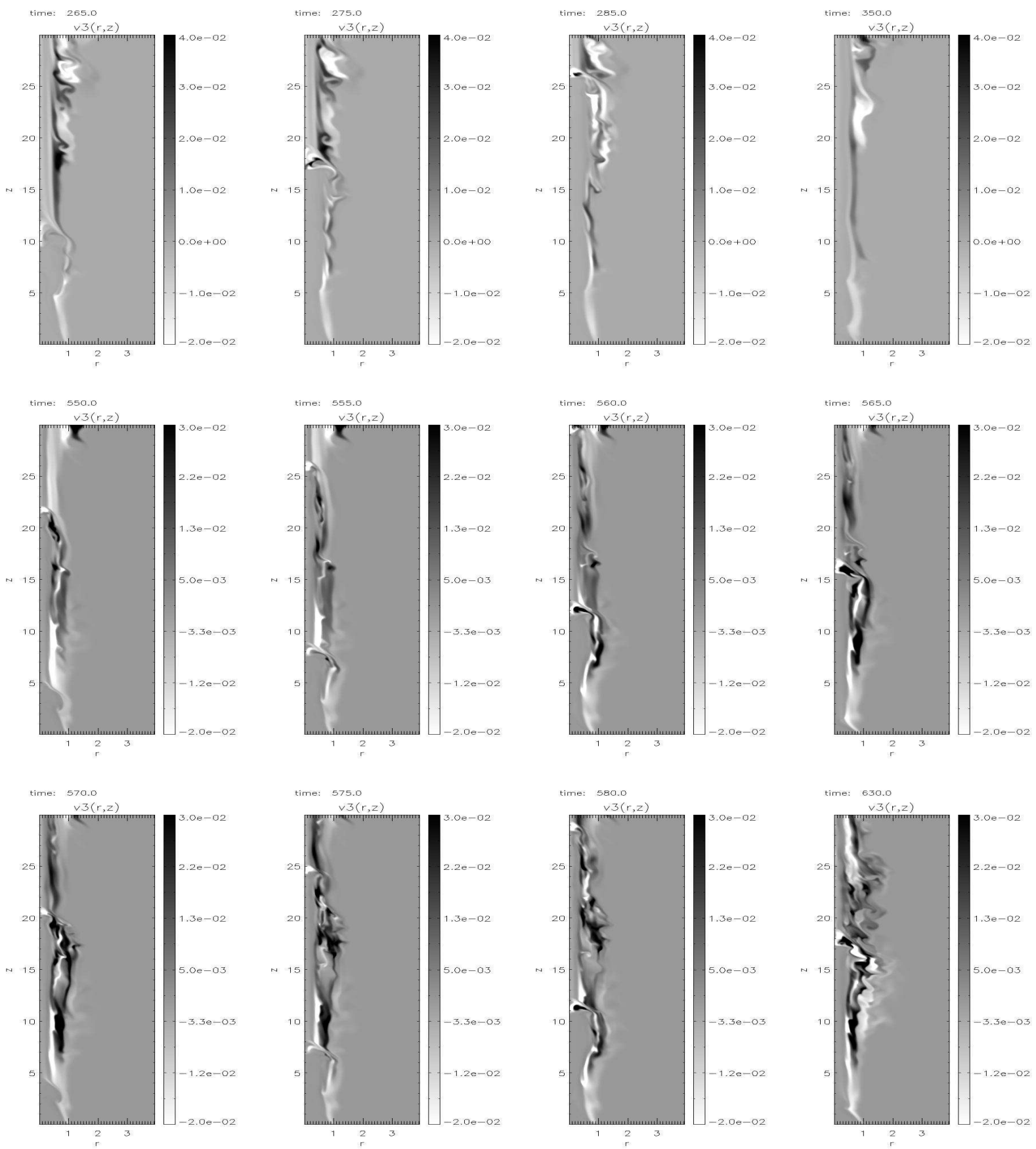

Figure 9. Axisymmetric simulation R16 continued with dynamical time steps $t=265,275,285,250,550,555,560,565,570,575,580,630$.

O'Neill, S.M., Tregillis, I.L., Jones, T.W., Ryu D., ApJ, 633, 717

Ouyed, R., \& Pudritz, R.E., 1997, ApJ, 482, 712

Ouyed, R., Clarke, D.A., Pudritz, R.E. 2003, ApJ, 582, 292

Pelletier, G., \& Pudritz, R.E. 1992, ApJ, 384, 117

Porth, O., \& Fendt, C. 2010, ApJ, 709, 1100

Pudritz, R.E., \& Norman, C.A. 1983, ApJ, 274, 677

Pudritz, R.E., Ouyed, R., Fendt, Ch., Brandenburg, A. 2007, in: B. Reipurth, D. Jewitt, \& K. Keil (eds.), Protostars \& Planets V,

University of Arizona Press, Tucson, 2007, p.277

Ramsey, J.P., Clarke, D.A. 2011, ApJ, 728, L11

Ray, T.P., Muxlow, T.W.B., Axon, D.J., Brown, A., Corcoran, D., Dyson, J., Mundt, R. 1997, Nature, 385, 415

Rieger, F.M. \& Mannheim, K. 2002, A\&A, 396, 833

Rieger, F.M. \& Duffy, P. 2004, ApJ, 617, 155

Rosen, A., Hardee, P., Clarke, D.A., Johnson, A. 1999, ApJ, 510, 136

Ryu, D., Jones, T.W. 1995, ApJ, 442, 228

Ryu, D., Jones, T.W., Frank, A. 1995, ApJ, 452, 785 


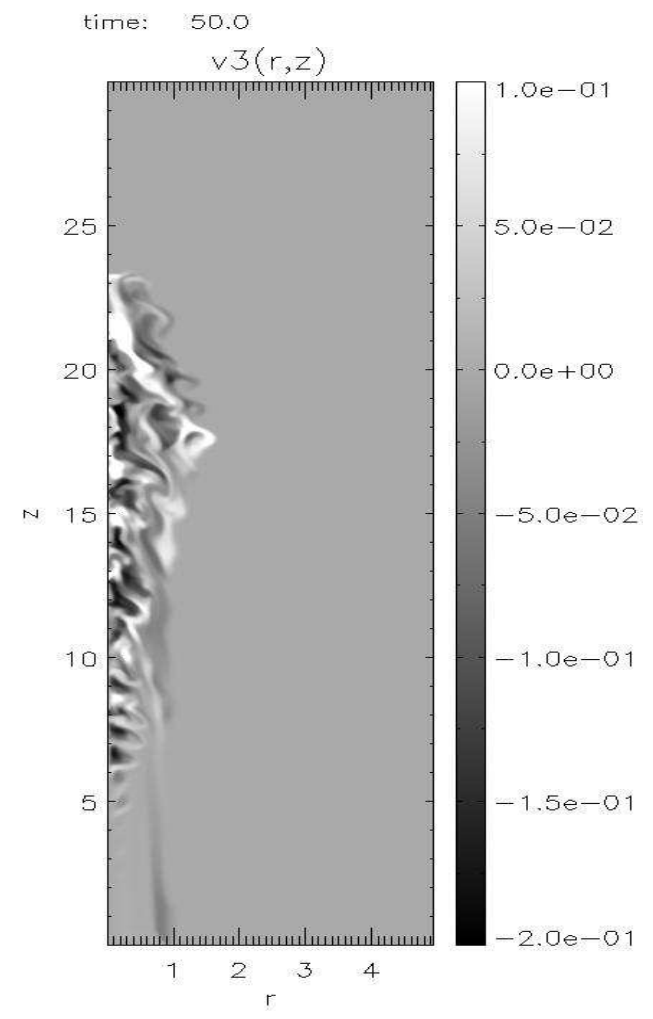

Figure 10. Toroidal velocity distribution of axisymmetric simulation run R19.

Soker, N. 2005, A\&A, 435, 125

Stone, J., Hardee, P. 2000, ApJ, 540, 192

Todo, Y., Uchida, Y., Sato, T., Rosner, R. 1992, PASJ, 44, 245

Todo, Y., Uchida, Y., Sato, T., Rosner, R. 1993, ApJ, 403, 164

Uchida, Y., Todo, Y., Rosner, R., Shibata, K. 1992, PASJ, 44, 227

Vitorino, B.F., Jatenco-Pereira, V., \& Opher, R. 2003, ApJ, 592, 332

Vlemmings, W.H.T. 2008, A\&A, 484, 773

Vlemmings, W.H.T., Surcis, G., Torstensson, K.J.E., van Langevelde, H.J. 2010, MNRAS, 404, 134

Wiseman, J., Wootten, A., Zinnecker, H., McCaughrean, M. 2001, ApJ, 550, L87

Woitas, J., Bacciotti, F., Ray, T.P., Marconi, A., Coffey, D., Eislöffel, J. 2005, A\&A, 432, 149

Zapata, L.A., Schmid-Burgk, J., Muders, D., Schilke, P., Menten, K., Guesten, R. 2009, A\&A, 510, 2 

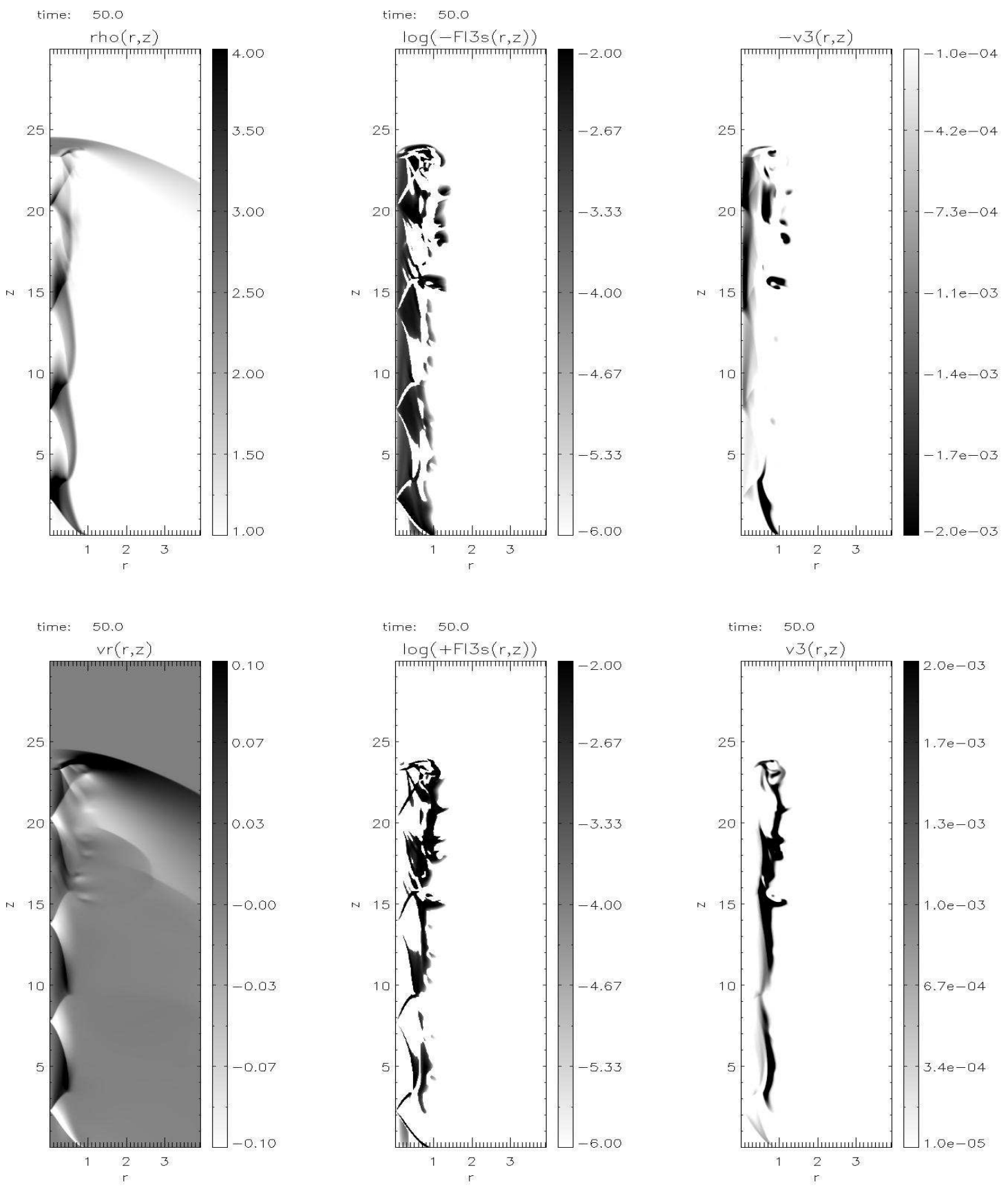

Figure 11. Axisymmetric simulation run R12. Shown are the density distribution (top left), the radial velocity distribution (lower left), the negative $(t o p)$ and positive (bottom) toroidal specific Lorentz force components (middle), and negative (top) and positive (bottom) toroidal velocity components (right) at dynamical time $t=50$. 


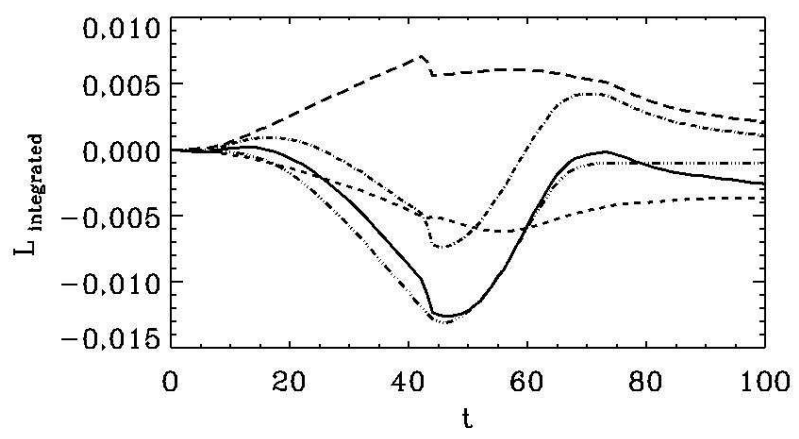

Figure 12. Time evolution of the domain-integrated angular momentum (a.m.) contributions for example simulation R01. Shown is the kinetic a.m. (positive contribution, thin dashed), kinetic a.m. (total contribution, thick dotted-dashed), magnetic a.m. (negative contribution, dashed), kinetic a.m. (negative contribution, thin dotted-dashed), and the total a.m. (solid line). Around time $t \simeq 45$ the jet terminal shock leaves the computational domain.

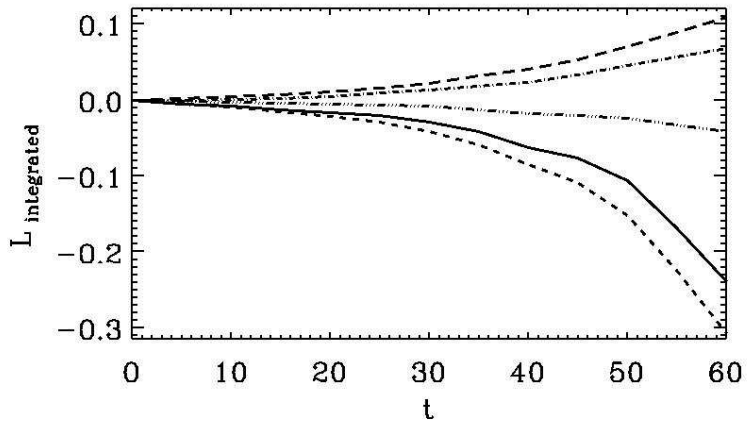

Figure 13. Time evolution of the domain-integrated angular momentum (a.m.) contributions for example simulation R12. Shown is the kinetic a.m. (positive contribution, thin dashed), kinetic a.m. (total contribution, thick dotted-dashed), magnetic a.m. (negative contribution, dashed), kinetic a.m. (negative contribution, thin dotted-dashed), and the total a.m. (solid line).
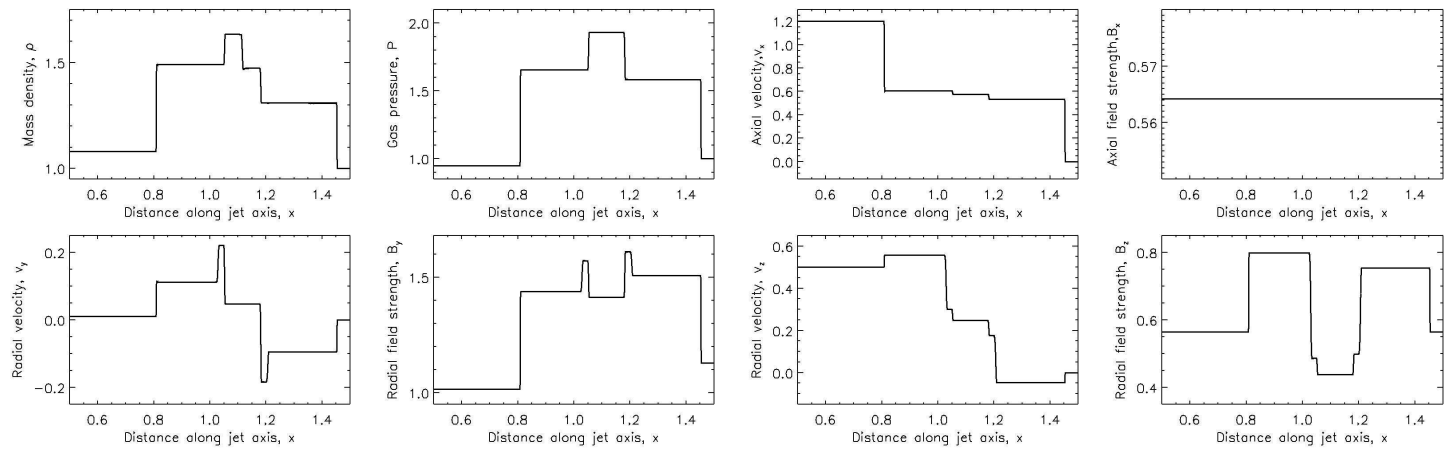

Figure 14. Simulation RJ11 in 1.5D Cartesian coordinates, equivalent to Fig. 2a of Ryu \& Jones (1995). Shown are: density; gas pressure; axial and radial velocities; axial and radial magnetic field strength (from top left to bottom right) at dynamical time $t=10$ (grid resolution 5000 cells per physical length 2.0). 

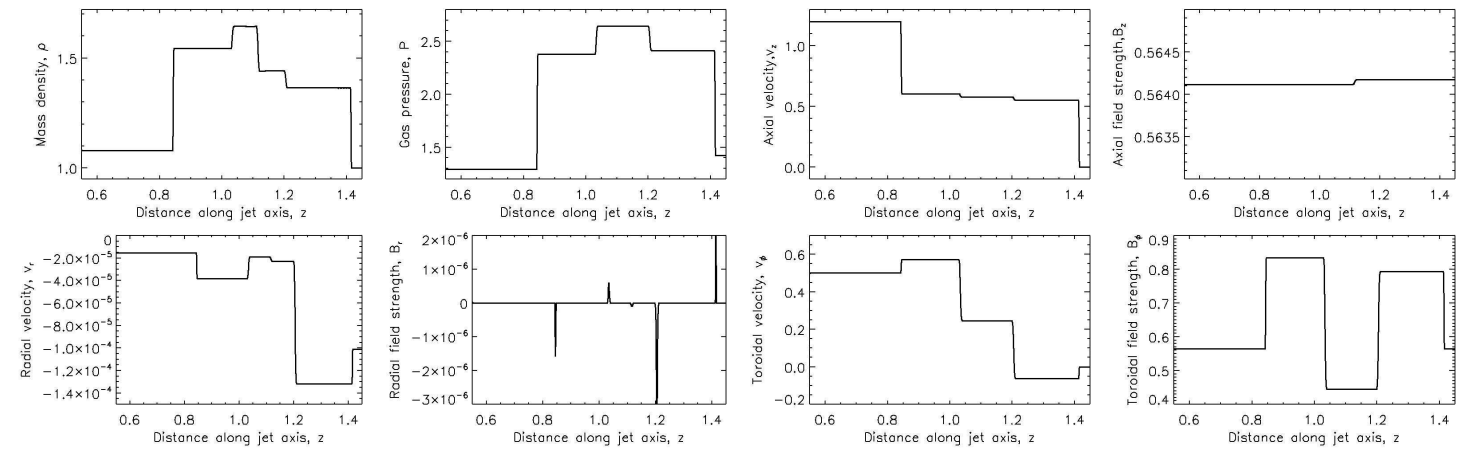

Figure 15. Simulation RJ09 in cylindrical coordinates and in axisymmetry, similar to Fig. 2a of Ryu \& Jones (1995). Only one of the perpendicular vector components - the toroidal component - is non-vanishing. Shown are: density; gas pressure; axial, radial, and toroidal velocities; axial, radial and toroidal magnetic field strength (from top left to bottom right) at dynamical time $t=10$ (grid resolution 3000 cells per physical length 2.0). 